\title{
3 Größe und Aktualität des Problems
}

\subsection{Daten: Wald und Bäume}

Es ist im Rahmen dieses Gutachtens nicht möglich, eine allumfassende und komplette Darstellung der Qualität der Gesundheitsversorgung in Deutschlandzu geben, ebensowenig wie hier ein Gesundheitssystemvergleich durchgeführt werden kann (vgl. Lauerer et al. 2011). Dieses Kapitel beschränkt sich vielmehr auf repräsentative Ausschnitte und legt dabei die Systematik der sechs Qualitätsperspektiven zugrunde, um die Thematik zu strukturieren und handhabbar zu machen.

Die bekannteste Systematik des Begriffs Qualität stammt von Donabedian (1986) und teilt die Qualitätsaspekte in Struktur-, Prozess- und Ergebnisqualität ein. Diese Systematik ist prägend und prägnant zugleich, allerdings hat sie den Nachteil der unvollständigen Abgrenzung zwischen Prozess- und Ergebnisqualität (s. Kap. 2.4.5), außerdem werden Patienten- und gesellschaftliche Perspektiven nicht genügend berücksichtigt. Ähnlich steht es auch um die zweite ebenso prägnante (und prägende) Systematik Over-, Under- und Misuse (Chassin et al. 1998), die als Über-, Unter- und Fehlversorgung ebenfalls vom Sachverständigenrat verwendet wurde (SVR 2001 Bd. III, S. 52ff.) und den Nutzen einer Behandlungs- und Untersuchungsmethode in den Mittelpunkt der Überlegungen stellt.

Es gab in der Vergangenheit mehrere Versuche, Aspekte wie Zugang, Effizienz und Patientenperspektive mit einzubeziehen (JCAHO 1991, IOM 2001). Done- 
bedian verwendet in seiner klassischen Arbeit „The Seven Pillars of Quality“ von 1990 nicht nur das Begriffspaar efficacy/effectiveness (absolute/relative Wirksamkeit), sondern auch efficiency/optimality (Effizienz/Optimalität; letzterer Begriff berücksichtigt neben dem punktuellen Verhältnis von Aufwand und Wirkung Abwägungen gegenüber anderen Verwendungsmöglichkeiten) und bezieht darüber hinaus die Angemessenheitskriterien acceptability (Akzeptabilität), legitimacy (Legitimität) und equity (zu übersetzen vielleicht am besten durch Fairness) mit ein (s. Abb. 19; Donabedian 1990; zum Begriff der Angemessenheit s. SVR 2008, Nr. 579). Im Methodenpapier 3.o des AQUA-Institutes wird auf die OECD Bezug genommen, die die Dimensionen Effektivität, Sicherheit und Patientenorientierung unterscheidet (AQUA-Institut 2013B, Arah et al. 2006). In der neuesten Fassung der DIN (DIN EN ISO 15224-2012) werden die (1) angemessene, richtige Versorgung, (2) Verfügbarkeit, (3) Kontinuität der Versorgung, (4) Wirksamkeit, (5) Effizienz, (6) Gleichheit, (7) Evidenz- bzw. Wissensbasierung, (8) Patientenorientierung, (9) Mitwirkung des Patienten, (10) Patientensicherheit und (11) Rechtzeitigkeit/Zugänglichkeit genannt.

Das vorliegende Gutachten legt das bereits in Kapitel 1.3 entwickelte Konzept der sechs Qualitätsperspektiven zugrunde, das sich in erster Linie an dem Erkenntnisinteresse orientiert. Neben der gesellschaftlichen sowie der Effizienzperspektive umfasst diese Cliederung die Patienten-, die professionelle, die institutionelle und die wissenschaftliche Perspektive des Qualitätsbegriffes (s. Abb. 20). Der Anlass, sich mit einer Erweiterung des Donabedian'schen

\section{The Seven Pillars of Quality}

- Efficacy: Ability of care, at its best, to improve health

- Effectiveness: The degree to which attainable health improvement is realized

- Efficiency: The ability to obtain the greatest health improvement at the lowest cost

- Optimality: The most advantageous balancing of costs and benefits

- Acceptability: Conformity to patients preferences regarding accessability, the patient-practicioner relation, the amenities, the effects of care, and the cost of care

- Legitimacy: Conformity to social preferences concerning all above

- Equity: $\quad$ Fairness in the distribution of care and its effects on health

Abb. 19 Systematik des Begriffs Qualität nach Donabedian 1990

- Gesellschaft: Population

- Nutzen: Allokation, Effizienz

- Patienten: Selbstbestimmung

- Professionen: Autonomie, Garantenstellung

- Institutionen: Organisation

- Wissenschaft: Deskription und Hypothesenbildung

Abb. 20 Die 6 Perspektiven des Qualitätsbegriffes, siehe Kapitel 1.3 
Konzeptes von $1986 \mathrm{zu}$ befassen, bestand für alle genannten Ansätze darin, dass die allgemeine Definition von Qualität (Erfüllung von Anforderungen) einen zu großen und beliebigen Definitionsspielraum bietet und es sinnvoll erscheint, für die Beschreibung des Status quo, vor allem aber für die Interpretation, Schwerpunktsetzung und auch für die Evaluation unterschiedlicher Interventionen ein inhaltlich besser fassbares und verbindlicheres Konzept zur Verfügung zu haben.

In der Schilderung des Status quo in diesem Kapitel wird also der sehr enge Horizont des Qualitätsbegriffes in der deutschen Diskussion erweitert. Es kann kein vernünftiger Zweifel bestehen, dass Nutzenaspekte (z.B. Wirksamkeit einer Therapie), Patienten- und gesellschaftliche Präferenzen wichtige qualitätsrelevante Parameter darstellen, das gleiche gilt für institutionelle Parameter, denn wenn eine qualitätsrelevante Leistung aus institutionellen Gründen (z.B. Innovationsresistenz, Ressourcenmangel) nicht umgesetzt werden kann, kommt es ebenfalls zu einem Qualitätsdefizit. Es soll hier nicht die Diskussion nochmals eröffnet werden, die in frühen Zeiten der Einführung der Evidence-Based Medicine von dem Standpunkt ausging, eine „gute“ Medizin sei eine solche, die nach EBM den höchsten Absicherungsgrad aufweist - hierbei handelte es sich um einen biomedizinischen Reduktionismus, der nicht sah, dass im randomisierten Versuch zwar eine Therapie als wirksam erwiesen sein kann, sie aber deswegen trotzdem noch nicht in der alltäglichen Umsetzung wirksam sein muss (sog. „letzte Meile“ der Versorgungsforschung, s. Pfaff 2003). Aber es bleibt festzuhalten, dass Nutzenaspekte und somit durch EBM abgesicherte Studienergebnisse von größter Bedeutung für die Qualität der Gesundheitsversorgung sind und somit neben die Aspekte der diagnoseund prozedurenbezogenen Behandlungsqualität (Komplikationen etc.) treten.

\section{Zusammenfassung:}

In Erweiterung der klassischen Qualitätssystematik nach Donabedian (1986, Struktur-, Prozess-, Ergebnisqualität) sind in der Vergangenheit mehrere inhaltlich deutlich prägnantere Qualitätssystematiken erarbeitet worden (zuletzt OECD, AQUA-Institut, DIN). Im vorliegenden Gutachten wird eine Systematik verwendet, die sich aus der Perspektive des Erkenntnisinteresses ableitet (s. Kap. 1.3).

\subsection{Qualitätsdefizite: gesellschaftliche Perspektive}

\subsubsection{Zugang zur Versorgung}

Der Zugang zur Gesundheitsversorgung (access) ist einer der zentralen Qualitätsaspekte und sorgt in den unterschiedlichen Gesundheitssystemen für intensive Diskussionen, die die jeweilige Struktur des Gesundheitssystems reflektieren. Man kann den Zugang zu Gesundheitsleistungen als Indikator für 
die Qualität der Versorgung einsetzen, typischerweise auf Ebene des gesamten Gesundheitssystems (SVR 2008, Nr. 491ff). Dieser Indikator hat eine geographische, eine soziale, eine Angebots- und eine Patientendimension. Erstere Dimension beschreibt die räumliche Erreichbarkeit, eine in den Augen der Bevölkerung sehr wichtige Eigenschaft (Zweifel 2007), die manchmal auch als trade off gegenüber der wohnortnah gebotenen Qualität in Erscheinung tritt (Wasem und Geraedts 2011; s. Kap. 4.2 zur Krankenhausplanung). Die soziale Dimension wird in diesem Gutachten z.B. bei der Diskussion möglicher unerwünschter Effekte von Pay for Performance ausführlich dargestellt, es geht hier um ethnische, geschlechtsspezifische und den sozialen Stand betreffende Faktoren. Letztlich kann auch ein exception reporting, wie es als Ausnahmeregelung im QOF-Programm in Großbritannien Eingang gefunden hat, hier eingeordnet werden (s. Kap. 6.4.2). Eine Zugangsproblematik ist natürlich vorhanden, wenn ein zu geringes Angebot von Gesundheitsleistungen vorliegt (mit queuing), oder wenn es patientenseitige Gründe gibt, die den Zugang einschränken.

In Deutschland ist die Zugangsproblematik im Vergleich zu den USA (aus Gründen des eingeschränkten Zugangs zu Versicherungen) oder Croßbritannien (s. Maurray 200o) gering ausgeprägt, der Zugang zum Gesundheitssystem ist aufgrund der Versicherungsstruktur grundsätzlich gut. Als Beispiel sei die Krankenhausversorgung genannt, für 97,5\% der Bevölkerung ist ein Krankenhaus innerhalb von 20 Minuten zu erreichen (Spangenberg 2012). Trotzdem ist es angebracht, diesen Indikator hier an den Anfang zu stellen. Wie aus der aktuellen Auseinandersetzung um die 4-Wochen-Frist für ein Facharzttermin hervorgeht - diese Thematik findet sich auch im Koalitionsvertrag der Großen Koalition vom 23.11.2013 wieder - gibt es für GKV-Versicherte ein Problem mit dem Zugang zur spezialfachärztlichen Versorgung im ambulanten Bereich. Bereits seit 10 Jahren erscheinen immer wieder Untersuchungen von verschiedenen Seiten, die - bei leider oft schlechter Qualität der Daten - zeigen, dass es für GKV-Versicherte zu weitaus längeren Wartezeiten als für Privatversicherte kommt, wenn sie einen solchen Facharzttermin brauchen. Diesem Befund ist natürlich widersprochen worden, trotzdem lässt die Zahl der Untersuchungen die Ergebnisse zumindest plausibel erscheinen, und der ökonomischen Plausibilität entspricht es auch. Eine Untersuchung ist wissenschaftlich publiziert worden (Lüngen et al. 2008), sie zeigte bei einer fingierten Terminvereinbarung bei 189 Arztpraxen Differenzen zugunsten von privatversicherten Patienten gegenüber GKV-Patienten von 17,6 Arbeitstagen bei Allergietestung mit Lungenfunktionsprüfung, 17,o Arbeitstagen bei Augenhintergrunduntersuchungen, 24,8 für eine Gastroskopie, 4,6 für einen Hörtest und 9,5 für ein NMR des Knies, insgesamt eine Verlängerung um den Faktor 3,08 (signifikant). In einer weiteren Untersuchung konnte nachgewiesen werden, dass eine Zunahme der Privatversicherten um ein Prozent im städtischen Umfeld eine Zunahme der Fachärzte um vier und im ländlichen Umfeld um drei Prozent zur Folge hat (Sundmacher und Ozegowski 2013). Diese Daten entsprechen in der Größenordnung anderen Untersuchungen der letzten Jahre und zeigen 
nicht unerhebliche Zugangsdefizite, die auf den Versicherungsstatus zurückzuführen sind.

Weitere Hinweise auf Probleme mit dem Zugang zu Gesundheitsleistungen zeigen vergleichende internationale Untersuchungen des Commonwealth Funds, an denen auch Deutschland teilgenommen hat (s. Abb. 21), und die aufgrund ihres longitudinalen Charakters als recht aussagekräftig angesehen werden können (Schoen et al. 2005, 2007, 2009, 2011). Die Wartezeiten vor jedwedem Arztkontakt waren länger als 5 Tage bei 13\%, 20\%, 26\% und 23\% der Befragten (in der Reihenfolge der Veröffentlichungen), auf einen sekundärfachärztlichen Termin von länger als 4 Wochen bei 22\% (2005) und 32\% (2009), und der Zugang war aus finanziellen Gründen erschwert bei 26\% (2009) und 22\% (2011) der Patienten.

In der Konsequenz muss konstatiert werden, dass in Deutschland der Zugangsproblematik größere Aufmerksamkeit zuteil werden muss, auch wenn aus historischen Gründen diese Problematik zunächst nicht relevant zu sein scheint. Ein nicht zu vernachlässigender Teil der Bevölkerung beobachtet solche Zugangsbeschränkungen in den letzten Jahren, und der Versicherungsaspekt spitzt die Problematik natürlich noch zu (s. auch Schoen et al. 2010).

Der Zugang zu Gesundheitsleistungen muss aber auch in die Planungskriterien aufgenommen werden, sowohl in der Krankenhausplanung als auch in der regionalen Planung des gesamten Versorgungsangebots (s. Kap. 4.2). Dies ist umso wichtiger, als dass die Verringerung der Zahl der Krankenhäuser durch die Verweildauerverkürzung und die Verlagerung von Leistungen in den ambulanten Bereich in Verbindung mit den anderen eine Zentralisierung bewirkenden Anreizen (z.B. Zentrenbildung, Mindestmengen etc.) die Proble-



Abb. 21 Vergleichende Patientenbefragungen in 6 (2005), 7 (2007, 2009) bzw. 8 (2011) Ländern zur Wartezeit auf jedweden Arzt $>5$ Tage, auf einen Facharzttermin $>4$ Wochen und finanziell erschwerte Inanspruchnahme („Kosten“); Daten hier für Deutschland in \% der Befragten (aus Schoen et al. 2005, 2007, 2009 und 2011, unterstützt vom Commonwealth Fund, Daten nicht zu allen Zeitpunkten vorhanden). 
matik der Erreichbarkeit in Zukunft wichtiger erscheinen lassen wird. Gleichzeitig wird die Bevölkerung älter und geht in manchen Regionen zurück (vgl. auch Gutachten des Sachverständigenrates „Koordination und Integration Gesundheitsversorgung in einer Gesellschaft des längeren Lebens “ [SVR 2009]), so dass sich auch aus dieser Sicht der Zugang zur Gesundheitsleistung als problematisch erweisen wird, denn die beiden Effekte treten misslicherweise in den gleichen Regionen (z.B. nordöstliche Bundesländer) auf. Es ist auch nicht in Stein gemeißelt, dass integrierte Versorgungsnetze, die wünschenswerterweise regionale Versorgungsstrukturen aufbauen, zumindest bei Übernahme der Versicherungsfunktion nicht auch eine Vorenthaltung von Leistungen in Betracht ziehen könnten. Ein weiterer Aspekt ist die Zugangsverschlechterung als Folge einer Malpractice Crisis durch Verlust der Haftpflichtversicherung, so wie wir es derzeit im Bereich der Hebammenversorgung erleben, und wie es z.B. in den USA bereits länger thematisiert wird (Dronove und Grona 2005, s. zusammenfassend Kap. 3.3.3.3).

\section{Zusammenfassung:}

Der Indikator „Zugang zur Versorgung“ hat eine geographische, eine soziale, eine Angebots- und eine Patientendimension. In Deutschland erscheint die Zugangsproblematik im Vergleich zu anderen Ländern zunächst als gering ausgeprägt. Allerdings geben in internationalen Vergleichsuntersuchungen 20 bis 30\% der Patienten an, sie hätten Wartezeiten auf einen Arztkontakt von mehr als 5 Tagen, speziell bei Facharztterminen, und der Zugang sei auch aus finanziellen Gründen erschwert. Bei GKV-Versicherten zeigt sich gegenüber Privatversicherten eine Verlängerung der Wartezeit auf einen Facharzttermin um den Faktor 3.

\subsubsection{Regionale Unterschiede in der Versorgung}

Wie im letzten Kapitel gezeigt, führt das Thema Zugang der Versorgung zur Thematik der Regionalität der Versorgung und deren Differenzen in Qualität und Kosten (Newhouse und Garber 2013). In einem kürzlich erschienenen Systematischen Review von 836 Studien zum Thema medical practice variation kamen 319 Studien aus den USA, gefolgt von Canada und Großbritannien, Deutschland lag mit 13 Studien (1,6\%) im Mittelfeld (Corallo et al. 2014).

Auch in Deutschland haben wir eine erhebliche Varianz von Kosten und Versorgungsqualität zwischen verschiedenen Bundesländern und auf der Ebene von kleinräumigen Versorgungsregionen wie Kreisen bzw. Mittelzentren (Lazitis et al. 2011), und zwar je nach Diagnose in einer Bandbreite um den Faktor 2 bis 4. Die Kostendifferenzen stehen in diesem Gutachten nicht im Mittelpunkt, es ist aber nicht von der Hand zu weisen, dass es sich bei solchen Unterschieden im Zugang von Leistungen, in der Zahl durchgeführter Leistungen und in der Behandlungsqualität jeweils um qualitätsrelevante Unter- 
schiede handelt. Wenn Patienten in einem Region einer 2- bis 4-fachen Häufigkeit einer Operation oder einem derart unterschiedlichen Risiko einer Komplikation ausgesetzt sind wie in einer anderen Region, wofür es keine Erklärung in der Krankheitshäufigkeit oder Morbidität gibt, dann muss sich die Frage stellen, ob es sich nicht ursächlich um Unterschiede in der Struktur oder der Ausführung der Versorgung handelt. Andererseits muss natürlich koinzidiert werden, dass in einer dezentral organisierten Gesundheitsversorgung es immer eine gewisse Streuung zwischen den Versorgern geben muss; rein aus statistischen Gründen ist nicht anzunehmen, dass alle beobachtbaren Parameter über eine große Versorgungslandschaft völlig gleich verteilt sind. Außerdem stehen nicht nur Systemtheoretiker auf dem Standpunkt, dass ohne eine gewisse Varianz keine Innovation möglich ist (Plsek und Wilson 2001B).

Bei der wissenschaftlichen Publikation, „mit der vor 40 Jahren alles anfing“, von Wennberg und Gittelsohn (1973) in Science, fällt zunächst auf, dass am Anfang dieses Artikel eine ausführliche Schilderung der Gründe für solche Untersuchungen gegeben wird: das Social Security Act von 1972, das über die Professional Standard Review Organizations (PSRO's) Standards einzuführen versprach und die Qualität der Versorgung verbessern sollte. Die Analyse selbst war im wahrsten Sinne des Wortes kleinräumig: es ging nicht um die ganze USA, wie es heute in Zeiten des Dartmouth Atlas (Newhouse und Garber 2013) und anderer Untersuchungen der Fall ist (vgl. Jencks et al. 2003, McGlynn et al. 2003), sondern um den Bundesstaat Vermont, Nachbarstaat von New York, mit damals 444.00o Einwohnern und 19 Krankenhäusern, die sich in 13 hospital-areas zusammenfassen ließen. Die Unterschiede in der Durchführung von Operationen sind Geschichte: pro 10.00o Einwohnern wurden pro Jahr Tonsillektomien (Minimum/Maximum) in 13 und 151 Personen durchgeführt, Appendektomien 10/32, Cholezystektomien (Frauen) 17/57 und - das hat die Untersuchung so bekannt gemacht - Hysterektomien in 20 bzw. 6o Personen.

Diese Kleinräumigkeit ist für die Problematik der regionalen Unterschiede in der Versorgung charakteristisch. In Deutschland können kleinräumige Unterschiede in der maximalen Lebenserwartung (ab Alter o) von rund drei Jahren nachgewiesen werden, die weniger von der Dichte der medizinischen Versorgung als von sozioökonomischen Faktoren abhängen (Lazitis et al. 2011). Diese Unterschiede sind besonders deutlich, wenn man nur die vermeidbaren Sterbefälle (z.B. durch unterlassene Prävention oder durch Lebensweise wie z.B. Rauchen) betrachtet (Sundmacher et al. 2011). Die Gesundheitsversorgung in Deutschland weist Unterschiede in der Zahl der Hüftendoprothetik um den Faktor 2,6 auf (altersstandardisierte minimale Rate 120, maximale Rate Bayern 166,9 pro 100.ooo Einwohner über 5 Jahre) (Schäfer et al. 2012), Hysterektomien um den Faktor 4,4 (Geraedts und Malik 2012), in der Wirbelsäulenchirurgie um den Faktor 4,8 (Fürstenberg et al. 2012).

Im Qualitätsbericht des AQUA-Institutes für die externe Qualitätssicherung nach $₫ 137$ a SCB V aus dem Jahr 2013 war ebenfalls eine regionale Auswertung 
Tab. 3 Minimum-Maximum-Verteilung der Indikatoren/Parameter aus dem Qualitätsbericht des AQUA-Institutes 2013 für die bradykarden Herzrhythmusstörungen auf der Basis der Daten 2011, Diff.: Faktor zwischen Min.- und Max.Wert. D Deutschland, BW Baden-Württemberg, BAY Bayern, B Berlin, BB Brandenburg, HB Bremen, HH Hamburg, HE Hessen, MV Mecklenburg-Vorpommern, NS Niedersachsen, NRW Nordrhein-Westfalen, RP RheinlandPfalz, SL Saarland, S Sachsen, SA Sachsen-Anhalt, SH Schleswig-Holstein, TH Thüringen.

\begin{tabular}{l|crrr}
\hline Indikator/Parameter & Min. (\%) & D (\%) & Max. (\%) & Diff. \\
\hline $\begin{array}{l}\text { Bradykarde Herzrhythmusstörung, nicht } \\
\text { leitlinienkonforme Indikation für SM }\end{array}$ & $2,3 \mathrm{HE}$ & 3,9 & $5,2 \mathrm{TH}$ & 2,3 \\
\hline $\begin{array}{l}\text { Idem, nicht leitlinienkonforme Wahl } \\
\text { Herzschrittmacher (SM) }\end{array}$ & $2,0 \mathrm{SL}$ & 3,0 & $4,7 \mathrm{SA}$ & 2,4 \\
\hline \begin{tabular}{l} 
Idem, lange Eingriffsdauer Einkammer-SM \\
\hline Idem, lange Eingriffsdauer Zweikammer-SM
\end{tabular} & $6,2 \mathrm{SL}$ & 11,8 & $20,1 \mathrm{HB}$ & 3,2 \\
\hline Idem; lange Durchleuchtungszeit Einkammer & $3,5 \mathrm{TH}$ & 8,4 & $13,0 \mathrm{HB}$ & 2,4 \\
\hline Idem, lange Durchleuchtungszeit Zweikammer & $0,9 \mathrm{HB}$ & 2,4 & $3,4 \mathrm{BAY}$ & 3,8 \\
\hline Idem, Komplikationen chirurgisch & $0,5 \mathrm{TH}$ & 1,0 & $1,4 \mathrm{MV}$ & 3,8 \\
\hline Idem, Vorhofsondendislokation & $0,4 \mathrm{HH}$ & 1,0 & $1,5 \mathrm{MV}$ & 3,8 \\
\hline Idem, Ventrikelsondendislokation & $0,3 \mathrm{HB}$ & 0,7 & $1,2 \mathrm{SA}$ & 4 \\
\hline Idem, niedrige Signalamplitude Vorhof & $2,6 \mathrm{SL}$ & 7,8 & $10,4 \mathrm{BB}$ & 4 \\
\hline Idem, niedrige Signalamplitude Ventrikel & $0,5 \mathrm{BB}$ & 1,2 & $1,8 \mathrm{SH}$ & 3,6 \\
\hline
\end{tabular}

der dort erhobenen Parameter auf Bundeslandebene enthalten, und zwar für die Daten des Jahres 2010 und 2011. Es leitet sich aus den obigen Ausführungen zwar ab, dass die Analysenebene „Bundesland“ für das eigentliche Phänomen der Regionalität der Versorgungsqualität zu grob ist, trotzdem ist diese Auswertung bemerkenswert und wichtig, zeigt sich doch, dass wir in Deutschland Unterschiede nicht nur im Bereich der Inanspruchnahme, sondern auch im engeren Feld der Behandlungsqualität haben. Die Menge der Daten ist nicht einfach darzustellen, deswegen wird hier eine Gruppe von Indikatoren ausgewählt (bradykarde Herzrhythmusstörung) und die Indikatoren bzw. Messparameter mit ihrem jeweiligen Minimum, dem Bundesdurchschnitt und dem jeweiligen Maximum zusammenzufassend dargestellt (s. Tab. 3). Man erkennt bei allen Indikatoren eine Variabilität zwischen dem Bundesland mit dem niedrigsten und dem höchsten Wert zwischen 1,9 und 4.

\section{Zusammenfassung:}

Auch in Deutschland gibt es eine medical practice variation je nach Diagnose in einer Bandbreite um den Faktor 2 bis 4 . Die Zahl der Hüft-OPs schwankt um den Faktor 2,6, die der Hysterektomien um den Faktor 4,4 und die Zahl der Eingriffe in der Wirbelsäulenchirurgie um den Faktor 4,8. Im Bereich der bradykarden Herzrhythmusstörungen (Auswahl) schwanken nach den Angaben des AQUAQualitätsberichtes die Indikatoren bzw. Messparameter um den Faktor 1,9 bis 4 . 


\subsubsection{Mindestmenge}

Dieses Kapitel müsste eigentlich „Nicht-Beachtung des Zusammenhangs zwischen Leistungsvolumen und Ergebnis" heißen, es hat sich aber in Deutschland der Begriff Mindestmenge eingebürgert. Da das Gesundheitssystem durch die Nicht-Beachtung des genannten Zusammenhangs jedoch enorme Qualitätseinbußen hinnimmt, muss diese Thematik unter „Qualitätsdefizite unter gesellschaftlicher Perspektive“ aufgeführt werden.

Die Mindestmengenproblematik ist in mehrfacher Hinsicht bemerkenswert und charakteristisch für den Zustand der Qualitätssicherung in Deutschland:

- Für den Indikator der Mindestmenge gibt es einen ausdrücklichen gesetzlichen gesetzlicher Auftrag an den Gemeinsamen Bundesausschuss ( $\$ 137$ Abs. 3 Satz 1 Nr. 2 und Satz 2 SGB V). Jedoch zeigen neuere Studien, dass die bestehenden Mindestmengen in Deutschland bisher nicht zu einer Veränderung der Leistungsstruktur geführt haben (De Cruppé et al. 2014, Peschke et al. 2014).

- Es handelt sich bei der Leistungsmenge um einen typischen Qualitätsindikator, der voraussagt, dass bei niedriger Leistungsmenge das Risiko zu schlechter Qualität zunimmt. Dieser Zusammenhang ist wissenschaftlich unbestritten und für viele Krankheitsbilder und Eingriffe belegt (vgl. z.B. Ross et al. 2010). Wie in Kapitel 2.4.2 ausführlich dargelegt, gibt es aber einen Überlappungsbereich, in dem dieser Indikator Einrichtungen als auffällig kennzeichnet (weil er sehr sensitiv eingestellt ist), die jedoch gar keine schlechte Qualität bieten - man möchte aber auf „Nummer sicher gehen“ und hat daher die Grenzwerte so gewählt, dass alle wirklich schlechten Einrichtungen von dem Indikator erkannt werden (s. Abb. 12). Man kann diese unausweichliche Eigenschaft von Indikatoren „intrinsische Ungerechtigkeit“ nennen, denn eine zugleich 100\%ige Sensitivität und Spezifität ist nicht darstellbar (s. Kap. 2.4.2). Diese Festlegung geschieht aber zum Schutz der Patienten, denn wenn man Einrichtungen mit schlechter Qualität erkennen kann, sollte man es auch tun - dieses Ziel einer patientenorientierten Qualitätssicherung ist einer anbieterorientierten Vorgehensweise vorgeordnet (s. „fünf Ziele“ in Kap. 1.4).

- Die Problematik dieses Indikators besteht nun darin, dass es nur wenig wissenschaftliche Hinweise darauf gibt, wo genau die Grenzwerte liegen (bei 20 oder bei 25 Operationen pro Jahr?). Dies hat auch wiederum ein Kurzreview des IQWiC aus dem Jahr 2012 belegt (IQWiG 2012). Wenn man die organisationstheoretischen Zusammenhänge mit einbezieht, die erklären, wie es zu der Qualitätsverbesserung kommt (es handelt sich nicht nur um einfache Skaleneffekte, sondern es kommen z.B. Teambildung und Vernetzung der Experten vor Ort hinzu; s. Huesch 2011), dann ist es auch alles andere als verwunderlich, dass es keine „Schallmauer“ gibt, an der die Qualität sprunghaft besser bzw. schlechter wird. 
In der konkreten politischen Situation hat dies aber zu einer Blockade im Gemeinsamen Bundesausschuss geführt, mit der Konsequenz, dass es auch sozialrechtliche Urteile gab, die die Begründungen für die Grenzwerte (nicht für den grundsätzlichen Zusammenhang) als nicht hinreichend für den Ausschluss eines Krankenhauses von der Versorgung ansahen. Die Mindestmenge kann daher als paradigmatisches Beispiel für die Grenzen eines governance-Konzeptes im Gesundheitswesen angesehen werden: die korporatistischen Strukturen können nicht beauftragt werden, sich selbst Einschränkungen zu verordnen. Hier muss (hier müsste) eine politische bzw. gesetzliche Regelung erfolgen, will man nicht damit fortfahren, dass die Interessen der Institutionen über dem Recht der Patienten auf optimale Qualität stehen.

- Die ganze Angelegenheit ist umso tragischer, als die Mindestmengenindikatoren (sie müssen für jede Erkrankung extra spezifiziert werden) dringend im Zusammenhang mit der Versorgungsplanung gebraucht werden (s. Kap. 4.2). Mindestmengen stellen zusammen mit anderen Einflussfaktoren (z.B. Zentrenbildung, Fallpauschalierung, Rückgang der Investitionsmittel) starke Anreize zur Zentralisierung der Versorgung dar (speziell im Krankenhausbereich). Sie sind damit als natürliche counterparts zum Zugangsindikator anzusehen und werden ebenso wie intakte Zugangsindikatoren für eine Fortentwicklung der Krankenhaus- und Versorgungsplanung dringend gebraucht (s. Kap. 4.2).

Nicht nur in den USA, sondern auch in Deutschland gibt es mehr und mehr Untersuchungen, die einen z.T. sehr deutlichen Zusammenhang zwischen Leistungsmenge und Qualität unter Beweis stellen (z.B. perinatale Mortalität, s. Heller et al. 2002). In einer kürzlich erschienenen Studie des WIdO-Institutes, das bei knapp 150.ooo Patienten mit Hüftoperationen im Rahmen des QSR-Projektes (Qualitätssicherung durch Routinedaten [s. Kap. 2.4.6]) durchgeführt wurde, lagen die risikoadjustierte go Tage-Sterblichkeit, die Revisionsnotwendigkeit (365 Tage), die Komplikationen, die thromboembolische Ereignisse und die Femurfraktur (alle go Tage) in dem Fünftel der Krankenhäuser mit der niedrigsten Eingriffshäufigkeit (weniger als 45 Eingriffe pro Jahr) um ein Drittel (signifikant) höher als im Fünftel der Krankenhäusern mit mehr als 200 Eingriffen pro Jahr (s. Tab. 4).

Trotz dieser eindrucksvollen Daten ist es schwierig, eine Schätzung für die Größenordnung der Qualitätsdefizite anzugeben, die das deutsche Gesundheitssystem wegen der Nicht-Beachtung der Mindestmengenproblematik jährlich erleidet. Wenn man aber die Literatur im In- und Ausland zur Kenntnis nimmt, erscheint es zumindest glaubwürdig, mal darüber nachzudenken, dass eine Zunahme der Qualitätsdefizite um ein Drittel durchaus im Bereich des Möglichen erscheint. Natürlich ist das nur eine Annahme, die man sich aber vielleicht einmal durch den Kopf gehen lassen kann. Der Effekt ist im Großteil der Studien „statistisch signifikant“, also in seiner Größenordnung nicht von der Hand zu weisen. Aber letztlich ist hier der Gesetzgeber gefragt. 
3 Größe und Aktualität des Problems

Tab. 4 Odds Ratio (mit Konfidenzintervall) unerwünschter Ereignisse bei der Hüftendoprothetik zwischen dem Quintil mit der niedrigsten und der höchsten Eingriffszahl pro Jahr. Außer Revision beziehen sich alle Komplikationen auf eine Beobachtungszeit von 90 Tagen (aus Jeschke und Günster 2014)

\begin{tabular}{l|ccc}
\hline unerwünschtes Ereignis & $<45$ Eingriffe/J. & Konfidenzintervall & $>200$ Eingriffe/lahr \\
\hline Revision (365 Tg.) & 1,37 & $1,16-1,61$ & 1 \\
\hline chirurgische Komplikation & 1,24 & $1,04-1,47$ & 1 \\
\hline Thrombose/Lungenembolie & 1,30 & $1,17-1,92$ & 1 \\
\hline Femurfraktur & 1,64 & $1,22-2,21$ & 1 \\
\hline Sterblichkeit & 1,48 & $1,04-2,11$ & 1 \\
\hline
\end{tabular}

\section{Zusammenfassung:}

Trotz des gesetzlichen Auftrags nach $§ 137$ Abs. 3 Satz 1 Nr. 2 und Satz 2 SGB V ist die Umsetzung der Mindestmengenindikatoren durch den Gemeinsamen Bundesausschuss kaum vorangekommen, weil es zwar nicht an wissenschaftlicher Evidenz für einen grundsätzlichen Zusammenhang zwischen Leistungsmenge und Qualität fehlt, dafür aber die Grenzwerte wissenschaftlich schwer zu belegen sind. Daraus hat sich eine Kontroverse entwickelt, der die Selbstverwaltung (Gemeinsamer Bundesausschuss) nicht gewachsen ist. Es werden dadurch im deutschen Gesundheitswesen Qualitätsdefizite toleriert, die in der Größenordnung zwar schwer bezifferbar sind, aber ein erhebliches Ausmaß besitzen dürften (ein Drittel, so legen Studien nahe). Hinzu kommt, dass Mindestmengen auch für die Fortentwicklung der Krankenhaus- und Versorgungsplanung gebraucht werden. Die Mindestmengenproblematik hat die Grenzen des Selbstverwaltungskonzeptes klar aufgezeigt, die Politik ist gefragt und muss gesetzgeberisch einschreiten.

\subsubsection{Integration und Koordination}

Integration und Koordination sind gerade unter dem Aspekt des Vorherrschens chronischer und multipler Erkrankungen in einer alternden Gesellschaft besonders wichtige Qualitätsaspekte und stellen daher eine der drei Dimensionen des Orientierungsrahmens dar, wie er in Kapitel 1.4 entwickelt wurde. Besondere Wichtigkeit kommt dieser Dimension aus Patientenperspektive zu (s. Kap. 3.4.1, vgl. auch area-Indikatoren in Kap. 2.4.9). Aus gesellschaftlicher Warte betrachtet, fallen Integrationsdefizite vor allem in zweierlei Hinsicht an:

- als Defizite der Steuerbarkeit des Gesundheitswesens, bedingt durch die sektorale Perspektive der Leistungserbringer, und

- als finanzielle Verluste, die durch die mangelnde Abstimmung der Leistungen (Stichwort Doppeluntersuchungen) bedingt sind. 
Wie hoch diese finanziellen Verluste sind, ist schwer zu schätzen, in einer älteren Studie von McKinsey aus dem Jahr 2004 (McKinsey 2004) wird mit einer darauf zurückgehenden „Effizienzlücke“ von 13-25\% der Ausgaben im deutschen Gesundheitssystem gerechnet. In Studien des Commonwealth-Funds gaben $20 \%, 15 \%, 18 \%$ der befragten deutschen Patienten mit chronischen Erkrankungen an, dass bei ihnen eine Untersuchung gemacht worden sei, die bereits kurz zuvor an anderer Stelle durchgeführt worden war (Schoen et al. 2005, 2007 und 2009). 80\% der Krankenhäuser haben ein Überleitungsmanagement, aber nur $45 \%$ folgen den Vorgaben des Nationalen Expertenstandards „Entlassungsmanagement in der Pflege“ (SVR 2012).

In der Konsequenz sind die Steuerungsdefizite wahrscheinlich weitgehender als man zunächst annimmt. Wenn man Infektionen bzw. Erkrankungen mit MRSA nicht als „Hospitalismus“-Problem ansieht, sondern als Populationsproblem mit Zirkulation dieser Erreger über die Sektorengrenzen hinweg (von der ambulanten Versorgung in die stationäre Versorgung, weiter in die Rehabilitation und/oder Pflege, wieder ins Krankenhaus zurück, endlich wieder in den ambulanten Bereich usw., s. auch 3.2.5.), dann ist dieses Problem zweifelsfrei von gesellschaftlicher Relevanz - die Leistungserbringer sind jedoch in ihrer sektoralen Logik gefangen und investieren nicht in die Behebung des Gesamtproblems, es sei denn, man vergütet sie dafür gesondert.

\section{Zusammenfassung:}

Die Integrationsdefizite durch die Sektorierung des deutschen Gesundheitssystems fallen als Defizite in der Steuerbarkeit und als finanzielle Verluste in der Größenordnung von 13 bis 25\% des Gesamtbudgets an (Einschränkung: ältere Zahlen). Ein Sechstel der Patienten geben an, Untersuchungen seien kürzlich bereits an anderer Stelle durchgeführt worden. Das Überleitungsmanagement wird nur bei $45 \%$ der Krankenhäuser nach Expertenstandard durchgeführt.

\subsubsection{Nosokomiale Infektionen und Antibiotikaresistenz}

\subsubsection{Konzept: Infection Control}

Das Thema „Integration und Koordination“ des vorangehenden Kapitels führt direkt zu den nosokomialen Infektionen und Antibiotikaresistenzen. Natürlich kann man dieses Thema auch unter „Fehlversorgung“ und „Patientensicherheit" abhandeln, aber es gibt ein Faktum, das es sinnvoll, fast zwingend erscheinen lässt, dieses Thema hier unter „Gesellschaftliche Perspektive“ anzusprechen: Nosokomiale Infektionen und Antibiotikaresistenzen sind das einzige Qualitäts- und Sicherheitsproblem, das „ansteckend“, also zwischen Personen übertragbar ist. Wenn sich zwei kardiologische Patienten ein Zweibettzimmer im Krankenhaus teilen, dann spielt es für den einen Patienten zunächst keine Rolle, wenn sein Bettnachbar eine Komplikation (z.B. Nachblutung an der Punktionsstelle) der Herzkatheteruntersuchung erleidet. Falls 
dieser jedoch eine (bekannte oder unerkannte) Besiedlung oder gar Infektion durch einen mehrfachresistenten Erreger hat (z.B. MRSA), dann wird die Situation für ihn brenzlich, denn es besteht die Gefahr, dass er sich ebenfalls diesen antibiotikaresistenten Erreger zuzieht, mit allen Folgen, angefangen von der Isolierung bis hin zu lebensbedrohlichen Erkrankungen. Allerdings kommt nicht nur der „Nachbarpatient“ als Quelle in Frage, sondern auch das Krankenhauspersonal (vor allem bei mangelnder Händedesinfektion), die materielle Umgebung (Türgriffe etc.) und - außerhalb des Gesundheitswesens Erreger und Antibiotikaresistenzen z.B. aus der Landwirtschaft.

Nosokomiale Infektionen und Antibiotikaresistenzen sind also ein „ökologisches" Problem, sie haben mit der Umgebung zu tun. Früher hat man sie als „Krankenhausinfektionen“ bezeichnet, das ist aus heutiger Sicht jedoch zu eng. Wir müssen sie stattdessen heutzutage als regionale, populationsbezogene Probleme verstehen, denn

- sie betreffen alle Bereiche und Sektoren des Gesundheitssystems, der Krankenhauspatient wird z.B. in eine Pflegeeinrichtung verlegt, von dort in den ambulanten Bereich, von dort in die Reha - immer geht die Infektion mit;

- es ist eine zunehmende Bedeutung von Faktoren zu beachten, die außerhalb des Gesundheitssystems ihren Ursprung haben, z.B. Antibiotikaresistenzen durch die Tiermast, die über Mitarbeiter der Landwirtschaft oder die Nahrungsprodukte selbst (z.B. Auftauwasser von Hühnchen, über das bei der Nahrungszubereitung gesunde oder auch kranke Personen infiziert werden), ,ins Gesundheitssystem“ importiert werden.

Diese Entwicklung macht das Problem nosokomialer Infektionen und Antibiotikaresistenzen zu einer äußerst komplexen Angelegenheit. Man steht im Grunde vor zwei Problemen, einerseits der Übertragung von Bakterien (Erregern), andererseits der Ausbildung von Resistenzen gegen Antibiotika (die ebenfalls über Bakterien übertragen werden). Aus dieser Dualität lassen sich die modernen Konzepte der „Gegenwehr“ ableiten: einerseits muss man die Übertragung verhindern, andererseits die Ausbildung der Resistenz. Im Krankenhaus verwendet man daher Infection Control-Strategien, die einerseits von der „Hygienekommission“ koordiniert werden (Händedesinfektion, Isolierungsvorschriften, technische Hygiene der Anlagen etc.), andererseits (im typischen Fall) durch die Arzneimittelkommission, die Leitlinien für eine sinnvolle Antibiotikatherapie erstellt, die einer Resistenzbildung vorbeugt, indem Reserveantibiotika nur dann verwendet werden, wenn sie wirklich angezeigt sind (s. Abb. 22). Weiterhin braucht man Spezialisten, die Hilfestellung geben, denn wenn der Patient aus einem Nachbarland mit hohen Raten an Mehrfachresistenzen erst in der Notaufnahme angelangt ist, kann es schon zu spät sein, mit Abstrichen und Isolierung zu beginnen: eine Vorbereitung nach Anmeldung des Patienten wäre besser gewesen (Rapid Response Team, s. Abb. 22). Hier hat der lange Jahre geforderte und jetzt umgesetzte Aufbau 


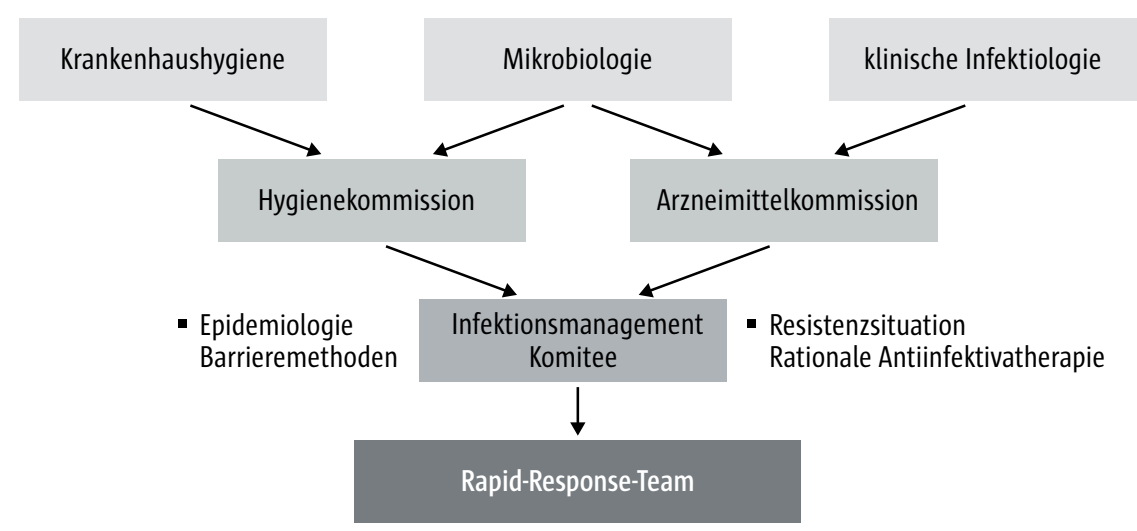

\section{Abb. 22 Infection Control Konzept, basierend auf der Dualität von Epidemiologie (Infektionsübertragung) und Steuerung der Antibiotikatherapie mit dem Ziel der Vermeidung von Resistenzen.}

eines Antibiotic Stewardship Programms, also der Ausbildung von Klinikern in der rationalen Therapie mit Antibiotika, sehr geholfen (s. Webseite der Deutschen Gesellschaft für Infektiologie, dgi-net.de).

Aber die Sache ist heute eben noch schwieriger geworden. Nosokomiale Infektionen und damit verbundene Resistenzen sind keine reinen Krankenhausprobleme mehr, sondern treten auf insgesamt vier Ebenen auf:

- auf der Ebene des einzelnen Patienten: Patienten können endogen, also durch die „mitgebrachte“ eigene Bakterienbesiedlung, und exogen, z.B. durch Krankenhauspersonal, infiziert werden;

- auf der Ebene der Institution (Leistungserbringer): Infektionen können hier sporadisch (z.B. weil ein Patient sie von außen mitgebracht hat), endemisch (ein Krankenhausmitarbeiter ist besiedelt und infiziert Patienten) und epidemisch auftreten („Ausbruch“, Übertragung von einem Patienten zum anderen z.B. über Tröpfcheninfektion wie bei Röteln);

- auf der Ebene des Gesundheitssystems: bestimmte Erreger werden zwischen den Sektoren bzw. Institutionen des Gesundheitssystems weitergegeben, die (wie in Deutschland) jedoch primär nur für den eigenen Bereich und nicht in Verantwortung für das ganze Gesundheitssystem handeln und daher die Qualität in erster Linie für den eigenen Sektor optimieren;

- auf der Ebene der Umwelt/Gesellschaft: in erster Linie wird die Tiermast und die Lebensmittelindustrie immer bedeutender, da sich dort mittlerweile wegen der unkontrollierten Gabe von Antibiotika ein großes Reservoir von resistenten Erregern gebildet hat. 


\section{Zusammenfassung:}

Nosokomiale Infektionen und Antibiotikaresistenzen sind das einzige Qualitätsund Sicherheitsproblem, das ansteckend ist. Moderne Infection Control-Konzepte umfassen die Verhinderung der Übertragung genauso wie die Vorbeugung durch eine rationale Gabe von Antibiotika, um Resistenzen zu vermeiden. Es handelt sich nicht um Krankenhausprobleme, sondern um „ökologische“ Probleme des gesamten Systems, und zwar auf der Ebene des einzelnen Patienten, der Leistungserbringer, des Gesundheitssystems und der Umwelt/Gesellschaft (z.B. Tiermast).

\subsubsection{Zahlen zu Infection Control: Zugang und Aussagekraft}

Man kann an dieser Stelle gar nicht genug betonen, wie sehr man im Bereich Qualität und Sicherheit von den Erfahrungen, Methoden und Vorgehensweisen aus der modernen Infektionsepidemiologie lernen kann. Diese Disziplin ist die erste gewesen, die sich explizit mit Qualität und Patientensicherheit beschäftigt hat, große Hindernisse zu überwinden hatte und ihre Methodik unter schwierigen Bedingungen entwickeln musste. Diese Vorbemerkung soll die Verdienste und die spezifischen Aufgaben der aktuellen Beschäftigung mit Qualität und Patientensicherheit nicht schmälern, insbesondere die Entwicklung der sog. generierenden Verfahren (z.B. CIRS; s. Kap. 2.2) im Rahmen moderner Sicherheitskonzepte, denn die Infektionsepidemiologie hatte immer dann ihre Schwierigkeiten, wenn unbekannte Faktoren (Unkown Unkowns, s. Kap. 2.1) nicht in ihre Modelle eingeschlossen werden konnten - weil man ihrer nicht gewahr war.

Die Infektionsepidemiologie hat aber Enormes geleistet in der Etablierung quantitativer Erfassungsmethoden (ausgehend von den surveillance-Definitionen der Centers of Disease Control) und in der Differenzierung zwischen Indikatoren (Vorhersage, Monitoring) und quantitativer Erfassung (s. Kap. 2.22.4). Insbesondere sei an dieser Stelle noch einmal herausgehoben, dass quantitative Verfahren weder klinische Diagnosen noch Indikatoren mit Monitoringfunktion darstellen. Die Infektionsepidemiologie verwendet genau spezifizierte Falldefinitionen, die

- allen Anforderungen hinsichtlich Reliabilität und Validität genügen müssen (Zuverlässigkeit z.B. bzgl. der Personen, die die Messung ausführen, und Validität bezüglich des Messzieles),

- einen optimalen Kompromiss zwischen Sensitivität und Spezifität aufweisen sollten (z.B. im Gegensatz zu Indikatoren, bei denen die Sensitivität im Vordergrund steht), und

- bei denen es sich um epidemiologische Falldefinitionen handelt und nicht um klinische Diagnosen, was zwar manchmal zu Differenzen mit den Klinikern führt, aber - das muss die Qualitätssicherung in Deutschland noch lernen - hinsichtlich der Reliabilität der Daten gerade im überinstitutionellen Vergleich unausweichlich ist (s. Einrichtung des „klinischen Vetos" [Talbot et al. 2013]). 
Infektionsepidemiologische Zahlen können aber, insbesondere in Zusammenhang mit Fragestellungen aus dem Bereich Qualität und Patientensicherheit, auch als Indikatoren verwendet werden, wenn sie Bereiche der Versorgung beschreiben und z.B. auf Komplikationen aufmerksam machen sollen (Vorhersagefunktion). So kann z.B. die Rate nosokomialer Wundinfektionen

- als quantitative Zahl genau die Häufigkeit dieser Komplikationen beschreiben,

- als Indikator die Qualität im Bereich der operativen Medizin einer Einrichtung einem Monitoring zugänglich machen,

n als Patientensicherheitsindikator (Qualitätsindikator spezieller Ausprägung) Aussagen über die Sicherheit in dem betreffenden Bereich treffen, oder

- als area-Indikator (s. Kap. 2.4.9) Aussagen über nosokomiale Infektionen und Antibiotikaresistenzen sowie - weitergehend - über die Integration und Koordination der Gesundheitsversorgung machen.

Gerade der letzte Punkt ist wichtig, wenn man den „ökologischen Zusammenhang“ betrachtet, in dem nosokomiale Infektionen und Antibiotikaresistenzen zu sehen sind (s. Kap. 3.2.5.1). Eine Region, die von einem Integrationsnetz versorgt wird, sollte durchaus auch danach beurteilt werden, wie hoch die Rate von Resistenzen oder die Rate von nosokomialen Infektionen ist - ein hervorragender Marker für die Kooperation der verschiedenen Einrichtungen und der Koordination zwischen Gesundheitssystem und anderen Bereichen der Gesellschaft wie der Nahrungsmittelindustrie.

\section{Zusammenfassung:}

Infektionsepidemiologische Methoden sind sehr weit entwickelt und stehen exemplarisch für quantitative Erfassungsmethoden (s. Kap. 2.3). Daten über nosokomiale Infektionen und Resistenzen können aber auch als Indikatoren verwendet werden (Vorhersagefunktion).

\subsubsection{Nosokomiale Infektionen in Deutschland}

Die internationale Literatur zu nosokomialen Infektionen und Antibiotikaresistenzen ist unübersehbar. Es sollen daher hier nur In aller Kürze die Fakten zur Situation in Deutschland wiedergegeben werden:

- Allein im Krankenhausbereich muss man mit zwischen 400.000 und 6oo.0oo nosokomialen Infektionen pro Jahr rechnen (Gastmeier et al. 2010). Es gibt zwei große Prävalenzuntersuchungen zur Situation in Deutschland, die im Jahr 1994 (Rueden et al. 1996) und 2011 (Behnke et al. 2013) durchgeführt wurden (NIDEP Studie I und II, „Nosokomiale Infektionen in Deutschland: Epidemiologie und Prävention“"). Die ältere Untersuchung umfasste 14.966 Patienten in 72 zufällig ausgewählten Krankenhäusern und ergab, dass 3,5\% der Patienten unter mindestens 
einer nosokomialen Infektion litten (einschließlich der bei Aufnahme schon bestehenden Infektionen), die jüngere im europäischen Rahmen durchgeführte Untersuchung an 9.626 Patienten aus 46 Krankenhäusern ergab, dass 3,4\% aller Patienten eine neu erworbene nosokomiale Infektion hatten $(5,1 \%$ aller Patienten einschließlich der Infektionen present on admission).

- Ungefähr ein Drittel der Infektionen dürfen als vermeidbar eingestuft werden, so dass also ca. $1 \%$ aller Krankenhauspatienten eine vermeidbare nosokomiale Infektion erleiden (zwischen 100.000 und 200.000 pro Jahr) (Gastmeier et al. 2010).

- So sehr in dem vorliegenden Gutachten die infektionsepidemiologischen Falldefinitionen (surveillance-Daten) und die daraus abgeleiteten Instrumente als Beispiel für das Funktionieren von klinischen Parametern für die Beschreibung von Qualität und Sicherheit befürwortet werden (s.o.), so sehr haben auch diese Grenzen. Das Problem besteht darin, dass (auch) surveillance-Daten die Problematik unterschätzen - allerdings in einem geringeren Maße als es z.B. administrative Daten tun (s.u.). Regelmäßig werden nämlich in klinischen und monozentrischen Studien weitaus höhere Werte gefunden, zuletzt in einer Studie an der Medizinischen Hochschule Hannover, wo prospektiv bei 11\% aller Patienten eine neu während des stationären Aufenthaltes entwickelte nosokomiale Infektion festgestellt wurde (Ott et al. 2013). In einer anderen Studie wurden bei $30.631 \mathrm{~Pa}-$ tienten in einem deutschen Schwerpunktkrankenhaus nach CDC-Kriterien eine Inzidenz von 5,3\% und nach zusätzlicher infektiologischer Beurteilung dann 8,4\% festgestellt (Hagel et al. 2013).

- Wie bereits angemerkt (s. auch Kap. 2.4.6) unterschätzen administrative Daten die Häufigkeit von Komplikationen und somit von nosokomialen Infektionen. In einer Studie in Deutschland werden durch Routinedaten weniger als die Hälfte der nosokomialen Infektionen identifiziert (Azaouagh und Stausberg 2008).

\section{Zusammenfassung:}

Allein im Krankenhausbereich muss man in Deutschland mit zwischen 400.000 und 600.000 nosokomialen Infektionen pro Jahr rechnen, ein Drittel davon sind als vermeidbar einzustufen. In der Konsequenz erleiden ca. $1 \%$ aller Krankenhauspatienten in Deutschland eine vermeidbare nosokomiale Infektion (zwischen 100.000 und 200.000 pro Jahr). Mehrere klinische und monozentrische Studien finden weitaus höhere Werte. Administrative Daten unterschätzen die Größenordnung um ca. 50\%.

\subsubsection{Antibiotikaresistenz in Deutschland}

Die Antibiotikaresistenz ist wahrscheinlich das wichtigste Qualitäts- und Sicherheitsproblem, das derzeit das Gesundheitswesen bedroht. Diese Aussage 
ist dadurch begründet, dass in den letzten Jahren Resistenzen aufgetreten sind, die keinerlei antibiotische Therapieoptionen mehr offen lassen, um die Patienten zu behandeln.

Was ist „Antibiotikaresistenz“? Mit diesem Begriff werden Eigenschaften von Bakterien beschrieben, die sie unempfindlich gegen Antibiotika machen. Die wichtigsten Mechanismen, die Bakterien hier einsetzen können (Auswahl), sind die Produktion von Enzymen, die die Antibiotika zerstören können (z.B. Betalaktamasen: Enzyme, die sog. Betalaktam-Antibiotika „unschädlich“ machen können, dazu gehört z.B. das Penicillin), die Veränderung der Kopplungsstellen an der Bakterienwand, mit denen sich die Antibiotika mit dem Bakterium verbinden, und die Veränderung der „Durchlässigkeit“ der Bakterienwand für das Antibiotikum. Resistenz ist durchaus ein natürlich vorkommendes Phänomen und wird relevant erst dann, wenn Bakterien mit einer dieser Eigenschaften über die anderen Bakterien ohne diese Eigenschaften die mengenmäßige Oberhand gewinnen können. Dies ist besonders bei schwer kranken Patienten, bei Patienten mit Verminderung der Immunabwehr (z.B. Alter) und bei Patienten, die in der letzten Zeit oder gleichzeitig viele Antibiotika verschrieben bekommen haben, der Fall.

Die Resistenzentwicklung gegen Antibiotika ist ein außerordentlich dynamischer Prozess, bei dem nicht vergessen werden darf, dass die Verfügbarkeit in einem Umfang, der eine klinische Anwendung erlaubte, noch keine 70 Jahre alt ist (Penicillin nach dem 2. Weltkrieg). Es ist zutreffend, die Resistenzentwicklung als klassischen komplexen Prozess im Sinne der Komplexitätstheorie zu bezeichnen (s. Kap. 8.3), der stark aus sich selbst heraus entwickelt und durch unvorhersehbare Entwicklungen gekennzeichnet ist. So sind z.B. der große Unterschied zwischen einigen Ländern (gerade typische Urlaubsländer mit Resistenzen gegenüber bestimmten Antibiotika von über $50 \%$ in dortigen Krankenhäusern) oder die Bedeutung der Antibiotika-Gabe in der Tiermast Phänomene, die man zuvor kaum als relevante Faktoren angesehen hatte. Man muss sich also genau überlegen, was die Angriffspunkte sind, an denen man mit einer räsonablen Aussicht auf Erfolg ansetzen kann. Da man weder Urlaubsreisen noch Immunschwäche oder das Älterwerden der Patienten unterbinden kann, bleibt in erster Linie der Versuch, mit Antibiotika kritisch und deeskalierend umzugehen.

In diesem Sinne kann das Problem der Antibiotikaresistenz nicht nur als komplexes Problem angesehen werden, sondern auch als Beispiel dafür, dass man in komplexen Systemen trotzdem eine gewissen Handlungskompetenz nicht zu verlieren braucht und nicht verlieren darf: festzuhalten ist, ohne Antibiotika gäbe es keine relevante Antibiotikaresistenz, und weiterhin ist der $\mathrm{Zu}$ sammenhang zwischen einem mengenmäßig hohen Verbrauch von Antibiotika und dem Entstehen einer relevanten Resistenz durch zahlreiche Studien über mehrere Jahrzehnte hinweg gut belegt (Auswahl: Bergman et al. 2009, Goossens et al. 2005, Steinke und Davey 2001). 
Die Häufigkeit multiresistenter Bakterien im Krankenhaus nimmt in Deutschland und Europa kontinuierlich zu, z.B. zwischen den Jahren 2001 und 2009 auf Intensivstationen in Deutschland von 4,5 auf knapp 8 multiresistenter Erreger in 1.00o Patiententagen (Mattner et al. 2012).

Die notwendigen Maßnahmen sind seit langem bekannt. Es geht in der ersten Linie um folgende Situationen:

- keine antibiotische Behandlung des grippalen Infekts, auch nicht der unkomplizierten Bronchitis (und Otitis media im Kindesalter),

- keine Fortführung der perioperativen Antibiotikaprophylaxe in der Chirurgie über die Dauer des Eingriffs hinaus,

- Antibiotikakonsil und regelmäßige Besprechung der mikrobiologischen Befunde (Resistenzstatistik) auf den Stationen mit hohem Antibiotikaeinsatz (z.B. onkologische und Intensivstationen), Einschränkung der empirischen Therapie mit Reserveantibiotika (ohne Vorliegen einer Resistenzbestimmung), Deeskalation der Therapie nach Vorliegen der Resistenzbestimmung auf das wirksame Antibiotikum mit dem engsten Spektrum,

- strengere Regulation des Antibiotikaeinsatzes in der Landwirtschaft, insbesondere Entkopplung von Antibiotikavertrieb und tierärztlicher Betreuung eines Betriebes (stattdessen Vertrieb der Antibiotika über Apotheken nach Rezeptur durch den Tierarzt, der langfristig an einen Betrieb gebunden ist).

Es bedarf hier kaum der Erwähnung, dass z.B. Punkt 1 und 2 längst in allen verfügbaren Leitlinien entsprechend niedergelegt und Gegenstand breit angelegter Interventionsstudien ist. In den Jahren 2008 und 2013 sind die Deutsche Antibiotikaresistenzstrategie Fassung I und II veröffentlicht worden (DART), in denen entsprechende Regelungen niedergelegt wurden. Wie in Kapitel 7.2 dargelegt, sind Regelungen wie regelmäßige interne Diskussion der Resistenzlage längst Bestandteil der gesetzlichen Vorschriften (Infektionsschutzgesetz $\$ 23$ ), werden aber ungenügend kontrolliert. Auch der landwirtschaftliche Gebrauch von Antibiotika ist im öffentlichen Diskus angekommen.

Trotz all dieser Aktivitäten bleibt der durchschlagende Erfolg bislang aus, die Bemühungen reichen offensichtlich nicht aus. Es sei hier nur ein einziges Bespiel herangezogen: die Weiterführung der perioperativen Antibiotikaprophylaxe im Krankenhaus über den Zeitraum des Eingriffes hinaus. Diese Maßnahme, nämlich die Gabe eines staphylokokkenwirksamen Antibiotikums zum Zeitpunkt des OP-Beginns (Hautschnitt) ist selbst ein „Klassiker“ des Infektions- bzw. Qualitätsmanagements, denn damit lassen sich postoperative Wundinfektionen gut verhindern. Der (zutreffende) Gedanke ist dabei, dass durch eine wirksame Antibiotikakonzentration im Blut und der Gewebsflüssigkeit im Bereich der OP-Wunde kleinste Mengen von Bakterien, die z.B. in der OP- oder Atemluft trotz aller Vorsichtsmaßnahmen noch vorhanden 
sind, daran gehindert werden, in den Stunden der Operation im Wundgebiet „Fuß zu fassen“, also Mikrokolonien zu bilden, die dann nach Wundverschluss weiterwachsen können. Die perioperative Antibiotikaprophylaxe ist gut untersucht und ihre Wirksamkeit z.B. bei Bauch- oder Knochenoperationen gut belegt. In den goer-Jahren des letzten Jahrhunderts war es noch so, dass diese Prophylaxe trotzdem oft nicht oder nicht zum richtigen Zeitpunkt durchgeführt wurde (Classen et al. 1992). Entsprechende Verbesserungsmaßnahmen waren aber meist erfolgreich, vor allem wenn das Pflegepersonal und die Anästhesie helfend eingriff (Wolters et al. 2000).

Als Schwierigkeit stellte sich jedoch heraus, dass die Operateure dazu übergingen, die Prophylaxe „zur Sicherheit“ nach der Operation mehrere Tage weiterzugeben, auch wenn der Patient keinerlei Infektionszeichen aufwies. Es gibt zu keinem Krankheitsbild eine Studie, die zeigt, dass dies auf den Outcome der Patienten irgendeinen positiven Einfluss hat; es steht allerdings außer Zweifel, dass die „Antibiotikalast“ eines Krankenhauses dadurch enorm ansteigt, was nicht nur die Folge hat, dass die Antibiotikakosten steigen, sondern vor allem dass sich die Resistenzsituation verschlechtert. Zahlreiche Empfehlungen und Initiativen versuchten diesem Missstand abzuhelfen.

Die oben bereits erwähnte NIDEP II-Studie (Behnke et al. 2013) erbrachte zu dieser Thematik jedoch nun aktuelle und erschütternde Daten, denn es wurden in dieser Studie nicht nur die nosokomialen Infektionen erfasst, sondern auch die Antibiotika, die zum Zeitpunkt der Datenerhebung gegeben wurden: $25,5 \%$ der Patienten erhielten Antibiotika, davon ein knappes Drittel $(28,5 \%)$ zur Prophylaxe, und wiederum davon fast die Hälfte $(47,6 \%)$ als perioperative Prophylaxe für einen Zeitraum länger als einen Tag. Hierzu wiederum eine überschlägige Abschätzung der Größenordnung des Problems:

- 10 Mill. stationär operierte Patienten pro Jahr in Deutschland,

- davon 25\% (2,5 Mill.) antibiotisch behandelt,

v davon 28\% (700.00o) wegen einer perioperativen Prophylaxe,

- davon die Hälfte (350.00o) länger als ein Tag,

- angenommene zusätzliche Dauer der Behandlung: 3 Tage,

- angenommene Tagestherapiekosten: $20 €$ einschließlich Verbrauchsmaterial und Arbeitszeit,

v zusätzliche Kosten pro Jahr: 20 Mill. €

Das ist nur eine Abschätzung, die die Größenordnung des Problems aufzeigen soll, aber wohlgemerkt: die sekundären Kosten für Patienten, die wegen Infektionen mit den konsekutiv vorhandenen resistenten Erregern behandelt werden müssen, sind hier gar nicht berücksichtigt. 


\section{NIDEP II-Studie in Deutschland}

Die perioperative Prophylaxe sollte nur während der OP gegeben werden. NI: nosokomiale Infektion (Behnke et al. 2013)

41.539 Patienten aus 132 Krankenhäusern

- bei $5,08 \%$ der Patienten war NI nachzuweisen

bei 3,76\% war die NI neu erworben

- 25,54\% der Patienten erhielten Antibiotika

davon $28,5 \%$ zur perioperativen Prophylaxe

- davon 47,6\% länger als ein Tag (1587 Pat.)

Für die Problematik der nosokomialen Infektionen und Antibiotikaresistenzen gelten also die gleichen Überlegungen wie für die anderen qualitäts- und sicherheitsrelevanten Überlegungen auch - daher wird in den Kapiteln 8 und 9 versucht, mit einem Rahmenkonzept dem Eindruck vorzubeugen, es ließe sich sowieso nichts mehr zum Guten wenden. Aber ein Sache muss an dieser Stelle doch noch angemerkt werden: es ist bzgl. der Antibiotikaresistenz sehr spät. Mehr und mehr breiten sich Bakterien aus, die neben Breitband-Betalaktamasen und anderen Resistenzmechanismen auch sog. Carbapenemasen aufweisen. Die Carbapeneme sind Antibiotika, die seit den goer-Jahren des letzten Jahrhundert sehr breit im Einsatz waren (und weiterhin sind) und als „Lieblingskind“ der Infektionsepidemiologen und Infection Control-Spezialisten zu gelten haben, denn es war vorhersehbar, dass die Situation sehr schwierig werden würde, wenn hier der Einsatz nicht vernünftiger erfolgt und entsprechend Resistenzen in großem Maßstab auftreten. Dies ist jetzt der Fall, es ist sozusagen das Fukushima der Antibiotikatherapie eingetreten. Patienten, die mit diesen Bakterien infiziert und erkrankt sind, haben keinerlei therapeutische Option mehr, sie sind wieder in die präantibiotische Phase angelangt, also in die erste Hälfte des 20. Jahrhunderts, zurückversetzt. Es wird der „Call for Cultural Change“ laut (Palmore und Henderson 2014), aber da die Neuentwicklung von Antibiotika ins Stocken gekommen ist, sieht die Sache schlecht aus. Fukushima der Antibiotikatherapie? - man könnte auch sagen: Fukushima der Krankenhausmedizin.

\section{Zusammenfassung:}

Die zunehmende Antibiotikaresistenz ist wahrscheinlich das wichtigste Qualitäts- und Sicherheitsproblem, das derzeit das Gesundheitswesen bedroht, insbesondere da sich die Anzeichen mehren, dass sich eine „totale“ Antibiotikaresistenz gegen alle verfügbaren Antibiotika ausbreitet. Die Häufigkeit multiresistenter Bakterien im Krankenhaus nimmt in Deutschland und international kontinuierlich zu. Die Resistenzentwicklung ist als klassischer komplexer Prozess zu bezeichnen, entsprechend vielschichtig sind die Interventionen. Die wichtigsten Angriffspunkte sind die Vermeidung der antibiotischen Behandlung des grippalen Infekts und der unkomplizierten Bronchitis, der Stop der Fortfüh- 
rung der perioperativen Antibiotikaprophylaxe in der Chirurgie über die Dauer des Eingriffs hinaus, die Einführung von Antibiotikakonsil und regelmäßiger Besprechung der mikrobiologischen Befunde (Resistenzstatistik) auf den Stationen mit hohem Antibiotikaeinsatz (bereits gesetzlich vorgeschrieben), und eine strengere Regulation des Antibiotikaeinsatzes in der Landwirtschaft mit Entkopplung von Antibiotikavertrieb und tierärztlicher Betreuung. Die Abschätzung der finanzielle Folgen auf der Basis der NIDEP II Studie in Deutschland ergibt, dass die zusätzlich auftretenden direkten Kosten allein der Fortführung der perioperativen Prophylaxe für einen Zeitraum länger als einen Tag im Bereich von 20 Mill. € liegen.

\subsection{Nutzen: Über-, Unter- und Fehlversorgung}

\subsection{1 Überversorgung}

Die Begriffe Über-, Unter- und Fehlversorgung wurden Ende der goer-Jahre in den USA entwickelt (Chassin 1998; Over-, Under- and Misuse) und mit dem SVRGutachten von 2001 in die deutsche Diskussion eingeführt, und zwar derart eindrucksvoll, dass sich aus den drei Begriffen schnell eine stehende Redewendung entwickelte. Über-, Unter- und Fehlversorgung rekurrieren auf den Nutzen einer Behandlungsmethode, genauer den Nettonutzen, denn eventuelle Risiken bzw. Schädigungen werden als „negativer Nutzen“ mit einbezogen. Das Konzept hat auch deshalb eine so große Bedeutung erlangt, weil es nahtlos mit dem Begriff des objektiven Bedarfs verbunden ist, der für Allokationsfragen grundlegende Bedeutung hat (s. Kap. 7.3). Die vom SVR vorgelegte Tabelle mit dem Zusammenhang zwischen der Art der Leistungserbringung auf der einen Seite und dem objektivem bzw. subjektivem Bedarf auf der anderen Seite sowie den resultierenden Formen der Über-, Unter- und Fehlversorgung ist hier noch einmal vorgelegt (SVR 2001, Nr. 40) (s. Tab. 5). Der Sachverständigenrat hat später den Begriff des objektiven Bedarfs durch Hinzunahme von Nutzenbestandteilen, die unter dem Terminus Angemessenheit zusammengefasst werden, ergänzt (SVR 2008, Nr. 579).

Überversorgung liegt demnach dann vor, wenn eine Leistung erbracht wird, die keinen Nettonutzen aufweist. Das IOM bezeichnet mit Überversorgung (overuse) eine Versorgungssituation, bei der das „potential for harm from the provision of a service exceeds the possible benefit" (Chassin et al. 1998). Der Sachverständigenrat definiert: Überversorgung sind „Versorgungsleistungen, die über die individuelle Bedarfsdeckung hinaus und ohne oder ohne hinreichend gesicherten gesundheitlichen (Zusatz-)Nutzen (z.B. aus Unwissenheit, Gefälligkeit, zu Marketingzwecken oder aus Einkommensinteressen) gewährt werden" (SVR 2001, Nr. 40).

Die Thematik der Überversorgung ist in der letzten Zeit durch die Choosing Wisely-Kampagne in den USA und Kanada nochmals in das Zentrum des Interesses gerückt (Morden et al. 2014, Levinson und Huynh 2014, Bericht in der Süd- 
Tab. 5 Über-, Unter- und Fehlversorgung und Bedarf (SVR 2001, Nr. 40)

\begin{tabular}{|c|c|c|c|}
\hline Bedarf ${ }^{\text {Leistung a) }}$ & $\begin{array}{l}\text { wird fachgerecht } \\
\text { erbracht }\end{array}$ & $\begin{array}{l}\text { wird nicht fach- } \\
\text { gerecht erbracht }\end{array}$ & wird nicht erbracht ${ }^{b)}$ \\
\hline $\begin{array}{l}\text { nur objektiver, } \\
\text { kein subjektiver Bedarf } \\
\text { (latenter Bedarf) }\end{array}$ & $\begin{array}{l}\text { bedarfsgerechte } \\
\text { Versorgung }\end{array}$ & Fehlversorgung & (latente) Unterversorgung \\
\hline $\begin{array}{l}\text { subjektiver und } \\
\text { objektiver Bedarf }\end{array}$ & $\begin{array}{l}\text { bedarfsgerechte } \\
\text { Versorgung }\end{array}$ & Fehlversorgung & $\begin{array}{l}\text { Unterversorgung } \\
\text { (ggf. Fehlversorgung) }\end{array}$ \\
\hline $\begin{array}{l}\text { nur subjektiver, kein } \\
\text { objektiver Bedarf }\end{array}$ & $\begin{array}{c}\text { Überversorgung } \\
\text { (ggf. Fehlversorgung) }\end{array}$ & $\begin{array}{l}\text { Überversorgung und } \\
\text { Fehlversorgung }\end{array}$ & $\begin{array}{l}\text { bedarfsgerechte } \\
\text { Versorgung }\end{array}$ \\
\hline
\end{tabular}

deutschen Zeitung vom 20.2.2014). Die Choosing Wisely-Kampagne, die im professionellen Umfeld in Deutschland noch nicht Fuß fassen konnte, nahm im Jahr 2012 durch einen Systematischen Review in einem der angesehensten Medizinjournale, den Archives of Internal Medicine, Fahrt auf: es wurden zum Thema Überversorgung gerade einmal 172 Artikel identifiziert, die nur 18 Therapie- und 24 diagnostische Verfahren betrafen (am häufigsten Antibiotika bei oberen Atemwegsinfektionen [Korenstein et al. 2012]). Die wissenschaftliche Bearbeitung des Themas Überversorgung ist also im äußersten Maße bruchstückhaft, was den Editor zu einem der kürzesten Editorial, der überhaupt denkbar ist, verleitete (ganze 9,5 Zeilen), das dem Titel: Overuse of Health Care. Where Are the Data? trug (Katz 2012).

Diese Initiative ist noch aus einem anderen Grunde bemerkenswert, denn und dies als Vorgriff auf die Diskussion des Professionalismus als Änderungsstrategie in Kapitel 9.4 - sie geht mit einem veränderten Konzept der ärztlichen Verantwortung einher, die entsprechende Textpassage sei daher hier wörtlich zitiert:

Die Thematik Überversorgung ist von breiter Relevanz. Mark Chassin hat vor einem Jahr in einem Artikel zum Thema „Improving the Quality of Health Care: What's Taking so Long?“ die fehlende Betonung des Themas Überversorgung als eines der wichtigsten Gründe genannt, warum die Qualitätsthematik sich so langsam entwickelt.

"Physicians' willingness to sincerely advance professionalism in medicine and to own their role asstewards of limited health care resources will be revealed by physician-led efforts such as Choosing Wisely. General acceptance of this effort to date by physicians and the public is encouraging and probably reflects our enduring trust in physicians as healers and credible leaders of health care reform. This trust must not be squandered; rather, it should be leveraged to restore balance in our nation's health care investment." 
Bezogen auf die deutschen Verhältnisse kann man folgende Bereiche heranziehen, die bzgl. Überversorgung eine besonders hohe Bedeutung haben:

- Anwendung von Verfahren, die aus unterschiedlichen Gründen nicht evaluiert sind oder bei denen in der Evaluation kein Nutzen festgestellt werden konnte, insbesondere im Bereich der sog. IGeL-Versorgung (,individuelle Gesundheitsleistungen“), die jenseits der GKV-Finanzierung den Patienten angeboten und von diesen frei finanziert werden müssen (Schnell-Inderst et al. 2011). Das Volumen dieses Marktes umfasste bereits im Jahr $2005 \mathrm{ca} .1 \mathrm{Mrd}$. $€$ (Zok und Schuldzinski 2005). Die meisten dieser Leistungen sind unbewiesener Natur und stellen eher eine Gefahr als ein Nutzen für den Patienten dar (Glaukom-Screening, Ultraschalluntersuchungen zum Screening des Ovarial-Ca., PSA zum Screening des Prostata-Ca etc.).

- im Besonderen die mangelnde Evaluation von medizintechnischen Verfahren, die erst in der letzten Zeit (endlich) eine gestiegene Aufmerksamkeit erhielten (z.B. Diskussionsforum von Gesundheitsforschungsrat und IQWiG 2012), denn eine Zulassung analog der Arzneimittel auf der Basis eines Nutzennachweises ist hier bislang nicht vorgesehen britischen Journalisten ist es kürzlich gelungen, eine nicht existente, fiktive Hüftendoprothese in der EU zuzulassen, was den Präsidenten der Deutschen Gesellschaft der Plastischen Chirurgen zu einem bitterbösen Artikel im Deutschen Ärzteblatt veranlasst hat (Vogt 2012).

- Indikationsausweitung von Verfahren, die grundsätzlich sinnvoll sind, aber in Deutschland in einem deutlich höheren Maße als in anderen Ländern durchgeführt werden, ohne dass dadurch ein zusätzlicher Nutzen entstehen würde (z.B. diagnostische Herzkatheteruntersuchungen). In besonderem Maß ist hier natürlich die DRG-getriebene Mengenausweitung vor allem operativer Verfahren zu nennen, die Zahl stationär behandelter Patienten ist von 16,5 Mill. Patienten im Jahr 2005 auf 18,6 Mill. Patienten im Jahr 2012 gestiegen (s. Kap. 9.2).

- Anwendung von Verfahren, bei denen die Studienergebnisse nicht vollständig publiziert werden können (sog. publication bias), weil die beteiligten pharmazeutischen Unternehmen die Daten nicht freigeben. Als besonders drastisches Beispiel sind hier Oseltamivir (Tamiflu) und Zanamivir (Relenza) gegen Influenza zu nennen, wo die Daten über Jahre nicht freigegeben wurden, obwohl (oder weil) sie die Unwirksamkeit der von öffentlichen Geldern in großen Mengen gekauften Medikamente bewiesen hätten (s. aktueller Cochrane-Review, Jefferson et al. 2014).

- Verschreibung von Medikamenten, die entweder als reine Me Too-Präparate (Nachahmerpräparate) einzuschätzen sind oder bei denen es bewährte Generika gibt (Einsparpotenzial für die GKV pro Jahr bis zu 2 Mrd. €, vgl. Arzneireport Barmer GEK, Gleaske und Schicktanz 2014). 
Die Liste ist nicht abschließend. Bekannt ist das Alles seit Jahrzehnten. Es wäre Zeit, dass sich auch in Deutschland einmal eine Choosing Wisely-Bewegung bildet, denn es ist ohne Zweifel so: die finanziellen Mittel, die hier verwendet werden, führen nicht nur in einem gewissen Teil der Patienten zu Schäden (sind also gleichzeitig Fehlversorgung), sondern stellen auch eine Verschwendung von finanziellen Mitteln dar, die an anderer Stelle so dringend fehlen.

\section{Zusammenfassung:}

Überversorgung sind Leistungen ohne Nettonutzen, oft mit Fehlversorgung verbunden. Die Thematik wurde durch die Choosing Wisely-Kampagne international wieder in das Zentrum der Diskussion gerückt. Es handelt sich um Verfahren, die aus unterschiedlichen Gründen nicht evaluiert sind oder bei denen in der Evaluation kein Nutzen festgestellt werden konnte (insbesondere im Bereich der sog. IGeL-Versorgung [,individuelle Gesundheitsleistungen“], jährliches Volumen von 1 bis 1,5 Mrd. €), mangelhaft evaluierte medizintechnische Verfahren (eine Zulassung analog der Arzneimittel auf der Basis eines Nutzennachweises ist hier bislang nicht vorgesehen), die Indikationsausweitung von Verfahren ohne zusätzlichen Nutzen (z.B. diagnostische Herzkatheteruntersuchungen und DRG-getriebene Mengenausweitungen), die Anwendung von Verfahren, bei denen die Studienergebnisse nicht vollständig publiziert wurden (als besonders drastisches Beispiel sind hier Oseltamivir [Tamiflu] und Zanamivir [Relenza] gegen Influenza zu nennen) und die Verschreibung von Medikamenten, die entweder als reine Me Too-Präparate (Nachahmerpräparate) einzuschätzen sind oder bei denen es bewährte Generika gibt (Einsparpotenzial für die GKV pro lahr bis zu 5 Mrd. €).

\subsubsection{Unterversorgung}

Unterversorgung? Man sollte glauben, dass Unterversorgung, also die Vorenthaltung von Leistungen mit bewiesenem Nettonutzen, in unserem angebotsorientierten und durch sektorale Mengenoptimierung getriebenen Gesundheitssystem keine Rolle spielen sollte. Das ist auch für viele Leistungen richtig, auf eine Hüftoperation braucht man (kaum) zu warten, die Notfallversorgung ist alles in allem gut organisiert, und es gibt kaum Zugangsbeschränkungen (zu den Ausnahmen s. Kap. 3.2.1).

Trotzdem haben wir in unserem Gesundheitssystem enorme Unterversorgungsprobleme, weil wir einige wissenschaftlich gut belegte Innovationen nicht oder nur sehr ungenügend umsetzen. Zwei Beispiele seien hier nur genannt, die Händedesinfektion als eine er wichtigsten Maßnahmen für die Prävention von Krankenhausinfektionen, und die adäquate Schmerztherapie als ein wichtiges Merkmal einer patientenorientierten Medizin und Prädiktor für einen positiven postoperativen Verlauf.

Unter Unterversorgung (underuse) versteht das IOM ein ,failure to provide a service that would have produced a favourable outcome for the patient" (Chassin et al. 1998). 
Der Sachverständigenrat bezieht den Begriff des Bedarfs mit ein: Unterversorgung sei „eine Versorgung bei individuellem, professionell und wissenschaftlich anerkanntem Bedarf, die verweigert wird oder nicht (zumutbar) erreichbar zur Verfügung gestellt wird, obwohl an sich Leistungen mit hinreichend gesichertem gesundheitlichen Nutzen und einer akzeptablen Nutzen-Kosten-Relation vorhanden sind“" (SVR 2001 Nr. 40).

Die entscheidenden Erkenntnisse zur Händedesinfektion (s. Kap. 2.4.2), allerdings damals noch mit sehr aggressiven Verfahren und noch nicht mit den heutigen alkoholischen Mitteln, datieren auf das Jahr 1847. Ignaz Semmelweiß, Geburtshelfer in München, stellt fest, dass die Sterblichkeit an Kindbettfieber, der postpartalen Sepsis, auf einer der beiden geburtshilflichen Stationen bei 20\% lag. Auf diesen Stationen waren Ärzte und Studenten tätig, die auch Leichen obduzierten. Auf der anderen Station, betreut durch Hebammen, lag die Rate der Todesfälle durch Kindsbettfieber jedoch nur bei 3\%. Er führte eine Händedesinfektion durch Chlorkalk ein, wodurch die Rate an Kindsbettfieber auf 1,2 bzw. 1,3\% abfiel, auf der ärztlich geführten Station also um den Faktor 20. Semmelweiß veröffentlichte erste Hygienevorschriften, wurde aber mit seinen Erkenntnissen nicht glücklich, er verstarb 1865 in $\mathrm{Bu}$ dapest.

Die Situation hat sich leider bis heute in Deutschland noch immer nicht durchschlagend gebessert. Wenn man davon ausgeht, und das ist Konsens praktisch aller Experten, die mit der Problematik langfristig zu tun haben, dass in Deutschland jährlich 7.500 bis 15.000 Todesfälle durch nosokomiale Infektionen im Krankenhaus auftreten und 30\% davon (vor allem durch Händedesinfektion) vermeidbar sind, dann heißt das, dass wir durch die Unterversorgung durch die Nichtanwendung dieser Behandlungsmethode „Händedesinfektion“ jährlich (mindestens, konservativ gerechnet) 2.500 bis 5.000 Todesfälle bei Patienten zulassen, die hätten verhindert werden können (Gastmeier et al. 2010). Man stelle sich vor dem inneren Auge vor, es gäbe ein Medikament, das 2.500 bis 5.000 Todesfälle in Deutschland jedes Jahr verhindern könnte, und wir würden dieses Medikament nicht geben - nicht weil es nicht verfügbar wäre, sondern weil wir es einfach nicht tun, was wäre dann in der öffentlichen Meinung (und auch berufs-, zivil- und strafrechtlich!) wohl los. Das Haftungsrecht arbeitet mit dem Begriff des Medizinischen Standards, gesetzt durch den Stand des medizinischen Wissens, das sollte man hier anfügen. Sehr optimistische Zahlen geben an, dass in 50\% der Situationen, in denen eine HD notwendig wäre (s.u. „Händedesinfektion [Aktion Saubere Hände]“), diese nicht durchgeführt wird. Keine Zeit? Nein, der wichtigste Faktor für diese Nicht-Anwendung ist die fehlende Vorbildfunktion (Pittet et al. 2004).

Diese kritische Betrachtung soll übrigens nicht den (partiellen) Erfolg und vor allem nicht den Einsatzwillen der vielen Personen im Gesundheitswesen schmälern, hier wirklich etwas zu ändern. Die Aktion Saubere Hände zum Beispiel, gegründet im Jahr 2007 durch das Robert Koch-Institut, die Gesell- 
schaft für Qualitätsmanagement in der Gesundheitsversorgung (GQMG) und das Aktionsbündnis Patientensicherheit, hat es erreicht, dass ein Drittel der Deutschen Krankenhäuser und viele Einrichtungen der benachbarten Sektoren sich aktiv um die Problematik kümmern. Genauso gibt es Aktivitäten, z.B. angestoßen durch die Universitätsklinik Münster, die große Fortschritte in Praxis und Wissen zu der Thematik erarbeitet haben. Es gibt viele aktive Einzelpersonen und auch Einrichtungen, die als top performer bezeichnet werden können, trotzdem - die große Wende lässt noch auf sich warten. Vermeidbare Todesfälle in der Größenordnung von 1.50o bis 4.500 jedes Jahr in Deutschland sind zu viel, für ein entwickeltes Gesellschafts- und Gesundheitssystem viel zu viel, man könnte auch sagen: eine Schande.

\section{Händedesinfektion (Aktion Saubere Hände)}

- vor und nach jedem Patientenkontakt mit direkter Hautberührung

- vor allen aseptischen Tätigkeiten z.B. Anschluss einer Infusion)

- nach Kontakt mit potenziell infektiösen Materialien

- nach Kontakt mit patientennahen Oberflächen oder Geräten

Ein anderes, ähnlich gelagertes Beispiel ist die Schmerztherapie. In der schon vielfach zitierten vergleichenden Befragung von Patienten in sieben westlichen Ländern in der Commonwealth Fund-Studie gaben 18\% der Patienten aus Deutschland an, sie hätten im Krankenhaus keine oder keine genügende Schmerztherapie erhalten (Schoen et al. 2005). In einer Evaluationsstudie der Initiative „Schmerzfreies Krankenhaus“ in Deutschland gaben 29,5\% der operierten und 36,8\% der konservativ behandelten Patienten an, in Ruhe moderate bis starke Schmerzen zu haben, 15 und 39\% der Patienten erhielten trotz Schmerzen keine Schmerztherapie, 55 bzw. 57\% gaben der Schmerztherapie schlechte Noten (Maier et al. 2010). In einer weiteren, prospektiven internationalen Studie in sieben europäischen Ländern (darunter Deutschland) ergaben Befragungen des Personals auf chirurgischen bzw. anästhesiologisch betreuten Stationen aus 746 Krankenhäusern, dass in 34\% keine Schmerzerfassung und in $56 \%$ keine Schmerzdokumentation vorgenommen wurde; nur $48 \%$ der Patienten waren präoperativ über die Schmerzen informiert worden (Benhamou et al. 2008).

Händedesinfektion und Schmerztherapie - beides sind Beispiele, die ja deswegen so große Bedeutung haben, weil sich jeder, der mit dem Cesundheitswesen in Berührung kommt, fragt, was er wohl selbst erleben wird, falls er bzw. sie vorhersehbarerweise einmal in die Situation kommt, dass er diese Unterversorgung wirklich erlebt. Manche mag als Unterversorgung empfinden, wenn er sein (teures) Originalpräparat nicht verschreiben bekommt und stattdessen mit Generika zufrieden sein muss. Aber im Vergleich dazu handelt es sich bei MRSA (einen mehrfach resistenten Krankenhauskeim) um ein ganz anderes Thema, nicht nur mit der Konsequenz einer deutlich erhöhten Sterb- 
lichkeit, sondern auch mit Isolierung, mit eingeschränkten Besuchsmöglichkeiten und mit der Chance, als MRSA-Träger entlassen zu werden und bei einer evtl. notwendigen Wiederaufnahme Schwierigkeiten zu haben, ein Krankenhaus zu finden, das einen trotz MRSA-Status behandelt.

\section{Zusammenfassung:}

Unter Unterversorgung versteht man die Vorenthaltung einer Behandlungsmethode mit nachgewiesenem Nutzen. Durch mangelnde Händedesinfektion, seit dem Jahr 1847 wissenschaftlich untersucht, versterben jährlich in Deutschland 2.500 bis 5.000 Patienten. Bei einer Befragung von Patienten in sieben westlichen Ländern gaben in einer Studie des Commonwealth Fund 18\% der Patienten aus Deutschland an, sie hätten im Krankenhaus keine oder keine genügende Schmerztherapie erhalten. In einer Studie der Initiative „Schmerzfreies Krankenhaus" gaben 29,5\% der operierten und 36,8\% der konservativ behandelten Patienten an, in Ruhe moderate bis starke Schmerzen zu haben, 15 und 39\% der Patienten erhielten trotz Schmerzen keine Schmerztherapie.

\subsubsection{Fehlversorgung und Patientensicherheit}

\subsubsection{Einführung}

Die meisten Formen der Fehlversorgung treten zusammen mit Überversorgung (eine Behandlungsmethode mit negativem Nettonutzen) und Unterversorgung (Vorenthaltung einer Behandlungsmethode mit positivem Nutzen und daher Verwirklichung eines Risikos) auf. Als Fehlversorgung (misuse) definiert das IOM ,avoidable complications that prevent patients from receiving full potential benefit of a service“" (Chassin et al. 1998). Der Sachverständigenrat schließt sich an: Fehlversorgung sei ,jede Versorgung, durch die ein vermeidbarer Schaden entsteht bzw. jede Versorgung mit Leistungen, deren Schaden oder Schadenspotenzial ihren (möglichen) Nutzen deutlich übersteigen“ (SVR 2001 Nr. 40). Dabei sind folgende drei Formen zu unterscheiden (ebd.):

- es handelt sich um „Leistungen, die an sich zwar bedarfsgerecht sind, d. h. weder Über- noch Unterversorgung darstellen und effizient sind, aber in der Form ihrer Anwendung den anerkannten fachlichen Qualitätskriterien ( $\$ \$ 106$ und 135-137 SGB V) nicht entsprechen und daher vermeidbare Risiken bzw. Schäden implizieren“,

- es handelt sich um eine „Überversorgung, die einen vermeidbaren Schaden bedeutet“, auch „wenn Überversorgung keinen positiven gesundheitlichen Nettozusatznutzen erzeugt, sondern ihre Risiken die anvisierten Nutzeneffekte übersteigen“, oder um

- „unterlassene, aber indizierte und an sich bedarfsgerechte Versorgungsleistungen (...), da entgangener Nutzen vermeidbaren Schaden bedeutet; Unterversorgung ist in diesem Sinne auch eine Fehlversorgung. “ 
Das Spektrum der Fehlversorgung ist groß, letztendlich können alle Formen von Komplikationen bzw. fehlerhaft erbrachten Leistungen, die im Qualitätsbericht zur externen Qualitätssicherung nach $\mathbb{} 137$ (AQUA 2013) verzeichnet sind, als solche angesehen werden. Im Krankenhaus-Report 2014 wurde von Geraedts eine Auswahl dieser Ereignisse zusammengestellt (Daten von 2011), die hier der Einfachheit halber zitiert werden (s. Tab. 6). Man erkennt schnell, dass es sich nicht um Einzelfälle, sondern um große Patientenzahlen handelt, bei denen eine Fehlversorgung besonders im Sinne des ersten Spiegelstrichs der SVR-Definition (s.o.) vorliegt.

Sicher sind nicht alle diese Komplikationen vermeidbar, trotzdem haben wir uns in gewissem Maße daran gewöhnt, diese Zahlen zu tolerieren. Wir rechnen Prozentsätze aus, die „Gnade der Rate“ sollte aber nicht davon ablenken, dass sich dahinter Einzelschicksale stecken - die eigentlich jeder einzeln abgeklärt werden müsste. Eine Wundinfektion tritt auf - was ist genau passiert? Auch die mangelnde Zentralisierung durch Nichtbeachtung der Abhängigkeit

\section{Tab. 6 Indikatoren im AQUA-Qualitätsbericht 2012 (Berichtsjahr 2011) mit „besonderem Handlungsbedarf" oder "Revisionsoperationen aufgrund von Komplikationen“, aus Geraedts 2014}

\begin{tabular}{|c|c|c|c|}
\hline Leistungsbereich & Indikator & $\begin{array}{c}\text { Anteil } \\
\text { Patienten }\end{array}$ & $\begin{array}{l}\text { Anzahl } \\
\text { Patienten }\end{array}$ \\
\hline $\begin{array}{l}\text { Herzschrittmacherrevision/System- } \\
\text { wechsel/Explantation }\end{array}$ & $\begin{array}{l}\text { Indikation zur Revision: } \\
\text { Sondenproblem }\end{array}$ & $3,3 \%$ & 3085 \\
\hline hüftgelenksnahe Femurfraktur & $\begin{array}{l}\text { Implantatfehllage, -dislokation } \\
\text { oder Fraktur }\end{array}$ & $1,1 \%$ & 1159 \\
\hline Idem & $\begin{array}{l}\text { Reoperation aufgrund von } \\
\text { Komplikationen }\end{array}$ & $3,0 \%$ & 3095 \\
\hline Hüftendoprothesen-Erstimplantation & Sterblichkeit im Krankenhaus & $0,2 \%$ & 293 \\
\hline Idem & $\begin{array}{l}\text { Reoperation aufgrund von } \\
\text { Komplikationen }\end{array}$ & $1,6 \%$ & 2572 \\
\hline $\begin{array}{l}\text { Hüftendoprothesen: Wechsel und } \\
\text { Komponentenwechsel }\end{array}$ & $\begin{array}{l}\text { Implantatfehllage, -dislokation } \\
\text { oder Fraktur }\end{array}$ & $1,9 \%$ & 486 \\
\hline Idem & $\begin{array}{l}\text { Reoperation aufgrund von } \\
\text { Komplikationen }\end{array}$ & $6,4 \%$ & 1612 \\
\hline $\begin{array}{l}\text { Knie-Totalendoprothesen- } \\
\text { Erstimplantation }\end{array}$ & Sterblichkeit im Krankenhaus & $0,1 \%$ & 137 \\
\hline Idem & $\begin{array}{l}\text { Reoperation aufgrund von } \\
\text { Komplikationen }\end{array}$ & $1,2 \%$ & 1803 \\
\hline $\begin{array}{l}\text { Knieendoprothesen: } \\
\text { Wechsel und Komponentenwechsel }\end{array}$ & $\begin{array}{l}\text { Reoperation aufgrund von } \\
\text { Komplikationen }\end{array}$ & $3,2 \%$ & 398 \\
\hline Cholezytektomie & $\begin{array}{l}\text { Reintervention aufgrund von } \\
\text { Komplikationen }\end{array}$ & $2,0 \%$ & 3441 \\
\hline
\end{tabular}


der Qualität vom Leistungsvolumen (Mindestmengen) erfüllt die Kriterien der Fehlversorgung (s. Kap. 3.2.3): im deutschen Gesundheitswesen hat man sich - auch wenn dies provokativ klingt - in der Suboptimierung eingerichtet, entscheidende Potenzial werde nicht gehoben.

Den o.g. Definitionen der Fehlversorgung ist der Bezug auf die medizinische Behandlung gemeinsam, ein Merkmal, das sich ebenso in den entsprechenden Definitionen im Bereich Patientensicherheit findet. Ohne die gesamte Nomenklatur an dieser Stelle wiederholen zu wollen (s. hierzu SVR 2007 Nr. 602 und Schrappe 2010C), sei hier nur die Definition des zentralen Begriffs des unerwünschten Ereignisses (UE) hervorgehoben, das drei Bedingungen genügen muss:

- das Ereignis ist negativ für den Patienten,

- es ist behandlungsbedingt (und nicht durch den Krankheitsverlauf), und

- es tritt unbeabsichtigt auf.

Ein vermeidbares unerwünschtes Ereignis (VUE) geht auf einen Fehler (Regelverletzung) zurück Wichtig ist weiterhin die Unterscheidung epidemiologischer Begriffe (wie z.B. das unerwünschte Ereignis) und juristischer Begriffe (z.B. der Behandlungsfehler, bei dem zusätzlich die mangelnde Sorgfalt entsprechend dem Dienstleistungsbegriff nachgewiesen werden kann) - der Schlüssel zur Auflösung von auf den ersten Blick widersprüchlichen Angaben zur Häufigkeit von unerwünschten Ereignissen (s. Kap. 3.3.3.2).

\section{Zusammenfassung:}

Fehlversorgung bezieht sich auf Behandlungsmethoden, bei denen ein vermeidbarer Schaden entsteht oder das Schadenspotenzial ihren Nutzen übersteigt. Komplikationen treten nach den Zahlen des AQUA-Qualitätsberichtes bei niedrigeinstelligen Prozentzahlen der Patienten auf und gehen damit in die Hunderttausende. Auch die mangelnde Zentralisierung durch Nichtbeachtung der Abhängigkeit der Qualität vom Leistungsvolumen (Mindestmengen) erfültt die Kriterien der Fehlversorgung.

\subsubsection{Häufigkeit von unerwünschten Ereignissen}

Um die Zahlen zur Häufigkeit von unerwünschten Ereignissen (UE), vermeidbaren unerwünschten Ereignissen (VUE) und (vermeidbaren) Todesfällen in Deutschland hat vor Kurzem noch einmal eine heftige Auseinandersetzung stattgefunden. Anlass war das Erscheinen des „Krankenhaus-Report 2014“, herausgegeben vom Wissenschaftlichen Institut der Ortskrankenkassen (WIdO), in dessen Einleitungskapitel die Zahlen des vom Aktionsbündnis Patientensicherheit (APS) angefertigten Systematischen Review aus dem Jahr 2008 (APS 2008) auf die heutigen Fallzahlen im Krankenhaus aktualisiert wurden (Geraedts 2014). 
Der Systematische Review wurde damals in der Gründungsphase des APS durchgeführt, um einen Ausgangspunkt für die Präventionsprojekte zu erhalten (s. Abb. 23), und wurde im Gutachten des Sachverständigenrates 2007 übernommen (SVR 2008, Nr. 604ff.). Die große Zahl der im ersten Review (Literatur bis 2005, 184 Studien; APS 2006) und der Aktualisierung (Literatur bis 2007, 241 Studien aus 44842 Publikationen; APS 2008, Lessing et al. 2010) identifizierten Studien und die Validität des Reviews (s.u.) wurde allgemein als Basis für die Entscheidung akzeptiert, mit der sofortigen Entwicklung von Präventionsprojekten zu beginnen und nicht noch eine sich über mehrere Jahre erstreckende neue epidemiologische Studie in Deutschland abzuwarten, vor allem weil aus Deutschland bereits 12 Studien in den Review eingeschlossen werden konnten.

Der erste Review wurde um einen Review zur Frage der Sterblichkeit ergänzt (APS 2007; s.u.). Es handelt sich um die größten Reviews in der internationalen Literatur. Eingeschlossen wurden Originalarbeiten aus den Datenbanken Pubmed und Embase, die in den Jahren 1995 bis 6/2007 publiziert wurden und Primärdaten zur Häufigkeit von unerwünschten Ereignissen, Schäden, Behandlungsschäden, Fehlern oder Beinaheschäden enthielten, die mit einer klar benannten Erhebungsmethode an einem definierten Patientenkollektiv durchgeführt wurden. Es wurden keine Studien eingeschlossen, die nur ein einzelnes Behandlungsverfahren oder ein einzelnes Medikament beschrieben, wohl aber Studien, die sich auf eine komplette Cruppe von Verfahren (z.B. alle unerwünschten Arzneimittelereignisse oder die Gesamtheit der nosokomialen Infektionen) bezogen. Die Literaturverzeichnisse der primär eingeschlossenen Studien wurden einer zusätzlichen Analyse unterzogen.

To Err Is

Human (IOM)



Vorber. Gründung APS

Syst. Review Epidemiologie I

Präventionsprojekte

Syst. Review

Epidemiologie II

Abb. 23 Zeitliche Einordnung der beiden Systematischen Reviews des Aktionsbündnisses Patientensicherheit (APS) in der Gründungsphase des APS (Gründung 2005). IOM Institute of Medicine, IfPS Institut für Patientensicherheit (Uni Bonn) 
Der größte Teil identifizierten Studien stammte aus den USA, Deutschland fand sich mit 12 Studien auf dem sechsten Platz. Die meisten der 241 Studien wurden in stationären Einrichtungen durchgeführt, ein weiterer Schwerpunkt lag im Arzneimittelbereich. Die Häufigkeit UE im Krankenhaus lag zwischen $0,1 \%$ und $20 \%$ und die Häufigkeit von VUE zwischen $0,1 \%$ und 10\% der eingeschlossenen Patienten. In Studien ab 1.00o Patienten stabilisiert sich die Häufigkeit von UE in einer Größenordnung zwischen 5\% und 10\% und die Häufigkeit von VUE auf Werte zwischen $2 \%$ und 4\% der eingeschlossenen Patienten. Diese Verteilung lässt indirekt auf eine gute Validität und Reliabilität der Studien schließen (s. Abb. 24), insbesondere da kein Zusammenhang zwischen Häufigkeit und Art der verwendeten Erhebungsmethode oder den Ländern, in denen die Studien durchgeführt wurden, festgestellt werden konnte.

Die deutschen Studien gaben Ereignishäufigkeiten wieder, wie sie auch in den internationalen Studien gemessen wurden. Das vorhandene Studienmaterial genügte weder, die Verteilung nach Fachdisziplinen zu bewerten, noch die verhältnismäßige Häufigkeit zwischen verschiedenen Ereigniskategorien (z.B. Medikationsereignisse oder Ereignisse im Bereich operativer Eingriffen) darzustellen. Die verwendete Erhebungsmethode wurde deutlich von der Art der Beobachtungsendpunkte bestimmt, so eignete sich die Durchsicht von Patientenunterlagen vor allem für die Feststellung von unerwünschten Ereig-

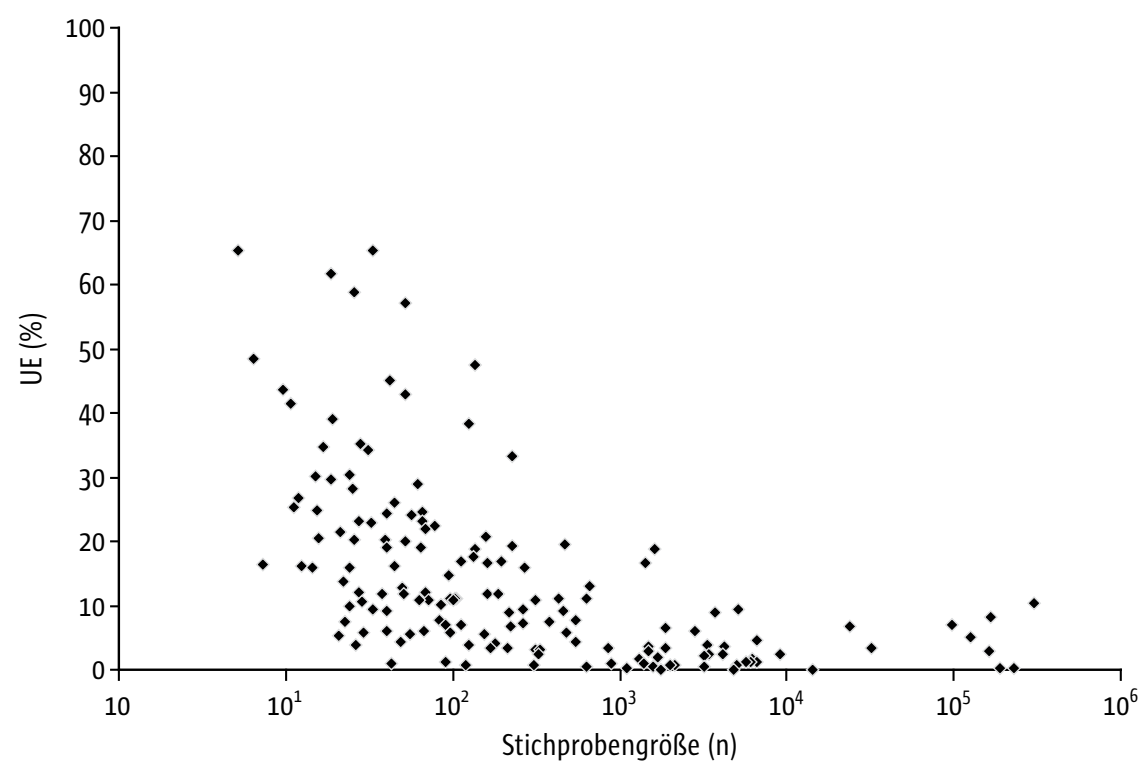

Abb. 24 Häufigkeit von unerwünschten Ereignissen (adverse events, AE) als Proportion (\% der Patienten mit mindestens einem Ereignis im Verhältnis zu allen Patienten) in Abhängigkeit von der Stichprobengröße der in die Untersuchung eingeschlossenen Patienten (modif. aus APS 2008). 
nissen und vermeidbaren UE, während Fehler und Beinaheschäden eher durch freiwillige Meldungen und direkte Beobachtung untersucht wurden.

Aus einer Untergruppe von 51 Studien des ersten Reviews wurden Daten zur Mortalität extrahiert (APS 2007). Es handelte sich um 39 Kohortenstudien und 12 Fallkontrollstudien. In 45/51 Studien wurde die Sterblichkeit bezogen auf die Grundeinheit der Gesamtpopulation untersucht, so dass Raten bzw. Proportionen zu bilden waren. In 43 Studien wurden Krankenhauspatienten beobachtet, 31 davon gehörten zu der wichtigen Cruppe der 36 Kohortenstudien über UE. Der mediane Stichprobenumfang betrug 4031 Patienten, 18 Studien wurden in den USA, 2 in Deutschland durchgeführt. Unerwünschte Ereignisse (UE) wurden in 43 und vermeidbare unerwünschte Ereignisse (VUE) in 19 Studien erhoben (11 beide). Die Ergebnisse der 19 Studien zu VUE als Todesursache zeigen, dass man mit einer Risikoangabe von o,1\% aller Krankenhauspatienten, an einem VUE zu versterben, die Situation sicher nicht übermäßig dramatisiert, sondern eher konservativ einschätzt (s. Abb. 25).

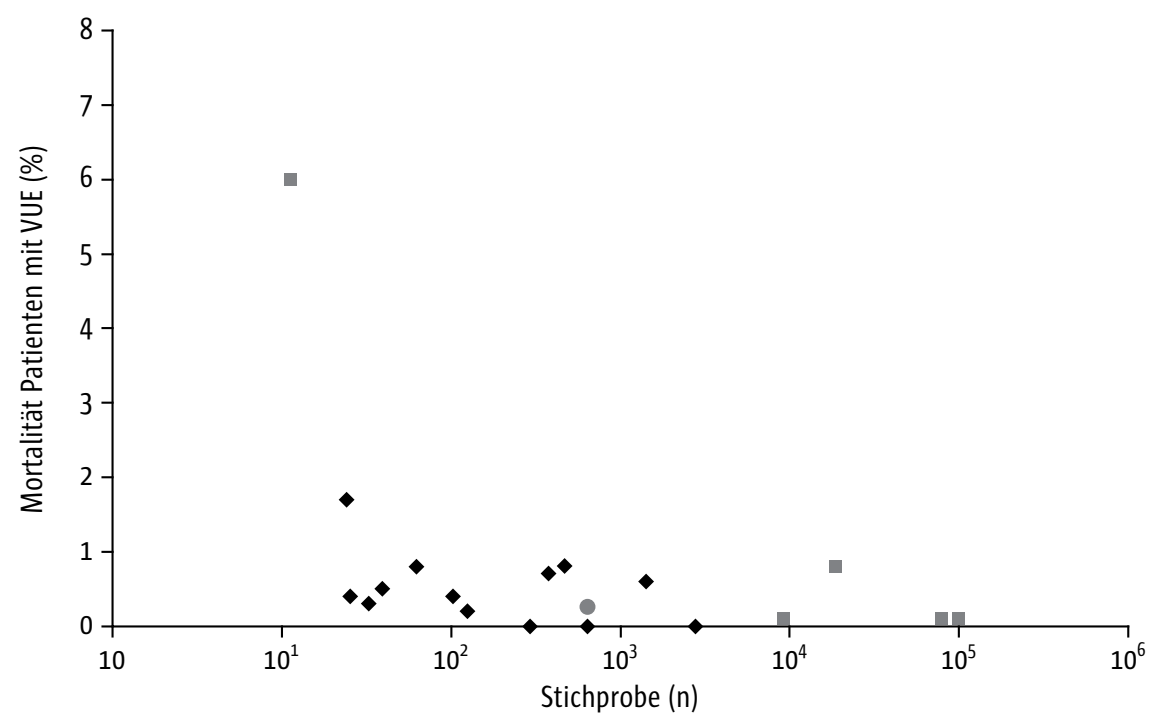

Abb. 25 Mortalität für Patienten an vermeidbaren unerwünschten Ereignissen (VUE) über alle Studiengruppen ( $n=19$ Studien). Halblogarithmische Darstellung über die Stichprobengröße. $(\bullet)=$ Kohortenstudien, bei denen ein kausaler Zusammenhang zwischen VUE und Sterblichkeit nachgewiesen wurde $(n=13),(\square)=$ Fallkontrollstudien an VUE: Verstorbene bezogen auf Verstorbene $(n=5)$ (wenig aussagekräftig), $(\bullet)=$ Fallkontrollstudie an VUE Verstorbene bezogen auf Gesamtpopulation $(n=1)$ (modif. nach APS 2007). 
Zusammenfassend kann aufgrund der Systematischen Reviews zumindest für den Krankenhausbereich von folgenden Größenordnungen ausgegangen werden:

- zwischen 5 und 10\% unerwünschte Ereignisse,

v zwischen 2 und 4\% vermeidbare unerwünschte Ereignisse,

- um $1 \%$ Behandlungsfehler und

n um o,1\% Todesfälle, die auf Fehler zurückgehen.

Bezogen auf rund 19 Mill. Krankenhausfälle pro Jahr in Deutschland (damals 17. Mill.) ergeben sich folglich in der Größenordnung

- 950.00o bis 1,9 Mio. unerwünschte Ereignisse,

[ 380.00o bis 760.00o vermeidbare unerwünschte Ereignisse,

- 190.00o Behandlungsfehler (juristisch mangelnde Sorgfalt) und

- 19.00o auf VUE zurückgehende Todesfälle.

Diese Zahlen wurde Anfang des Jahres 2014 u.a. von der Deutschen Krankenhausgesellschaft („verzerrte Darstellung“), dem Deutschen Ärzteblatt („subjektiv gefärbte Zahlenspielchen“, die bei der Bevölkerung „Ängste schüren“) und der Bundesärztekammer („uralte Metaanalysen aus den USA“, die alleine dazu dienten, „Stimmung gegen die Krankenhäuser zu machen“) in Zweifel gezogen. Zum einen wurde argumentiert, dass mittlerweile (z.B. durch Initiativen des Aktionsbündnis Patientensicherheit, an dem die genannten Institutionen sehr aktiv mitarbeiten) viele Präventionsmaßnahmen in Kraft gesetzt worden seien, so dass die Zahlen nicht (mehr) stimmen könnten - ein sehr optimistisches Argument, das voraussetzt, dass mit dem guten Dutzend an Maßnahmen, die in der letzten Zeit entwickelt worden sind, bereits ein turn around möglich gewesen ist. Zum anderen wird betont, dass durch die Schiedsstellen der Landesärztekammern ja nur einige Tausend Behandlungsfehler im Jahr begutachtet bzw. bestätigt werden konnten, so dass nicht knapp 20.00o Todesfälle auftreten könnten.

Hier muss nochmals darauf verwiesen werden, dass epidemiologische Zahlen, wie sie in den Studien zur Häufigkeit und in den Reviews verwendet werden, streng von Daten abgegrenzt werden müssen, die dem juristischen Umfeld entstammen, also aus den Schiedsstellen, den Haftpflichtversicherern und den Krankenkassen kommen. Man geht in Deutschland von ca. 20.00o Fällen mit nur 500-60o Todesfällen pro Jahr aus, die durch die Schiedsstellen oder juristisch weiter verfolgt werden. Es handelt sich dabei nicht um populationsbezogene Daten, und man kann nicht davon ausgehen, dass alle UE und VUE sowie Behandlungsfehler, die epidemiologisch nachweisbar wären, juristisch verfolgt werden. In der internationalen Literatur wird dieses Phänomen als litigation gap (übersetzt etwa „Haftungslücke“) bezeichnet (Blendon et al. 2002, Davis et al. 2002, Studdert et al. 2000): nur zwischen 1 und 6\% der Patienten, die ein Ereignis erleiden, beschreiten den Klageweg. In einer deutschen Studie zu vermeidbaren (!) nosokomialen Infektionen wurde nachgewiesen, dass sogar nur 
0,2\% der betroffenen Patienten das Ereignis juristisch klären lassen (Mönch et al. 2011). Korrigiert man die juristischen Daten um den Faktor 30, der sich aus einer Quote der juristischen Klärung von 3\% ergibt, kann man wieder auf die Größenordnung der epidemiologischen Daten rückschließen (s. Abb. 26).

Aber natürlich muss man sich um eine Plausibilisierung der o.g. Zahlen bemühen, insbesondere der Zahlen, die die vermeidbare Sterblichkeit betreffen. Drei Möglichkeiten sind dazu geeignet:

1. Vergleich mit den epidemiologischen Zahlen aus anderen Systematischen Reviews,

2. Vergleich mit epidemiologischen Zahlen aus anderen Datenquellen (z.B. nosokomiale Infektionen),

3. Vergleich mit Patientenbefragungen.

- Studien zur Häufigkeit von unerwünschten Ereignissen liegen seit mehr als 20 Jahren vor. Systematische Reviews zu diesen Studien sind in den letzten Jahren seltener durchgeführt worden, weil der Kenntnisstand allgemein als gegeben angesehen wird. Ein Systematischer Review aus dem Jahr 2008 zeigte bei knapp 75.0oo Krankenhauspatienten eine Häufigkeit von UE von 9,2\%, 43,5\% davon waren vermeidbar (VUE), die Sterblichkeit lag bei $7,4 \%$. Bezogen auf die VUE würde dies einer vermeidbaren Mortalität von über o,68\% entsprechen, somit einer Zahl, die deutlich über der Schätzung aus dem APS-Review von o,1\% liegt (de Vries et al. 2008). Ein Systematischer Review aus der Arbeitsgruppe der Forschergruppe um den bekannten Patientensicherheitsforscher Charles Vincent aus dem Jahr $2013 \mathrm{zu} 14$ Studien an 16.424 chirurgischen Patienten aus England kommt ebenfalls zu höheren Ergebnissen als das APS: 14,4\% erleiden ein UE, 5,2\% ein VUE und o, 52 versterben, bezogen auf die VUE also etwa $0,16 \%$ aller operierten Patienten (Anderson et al. 2013).

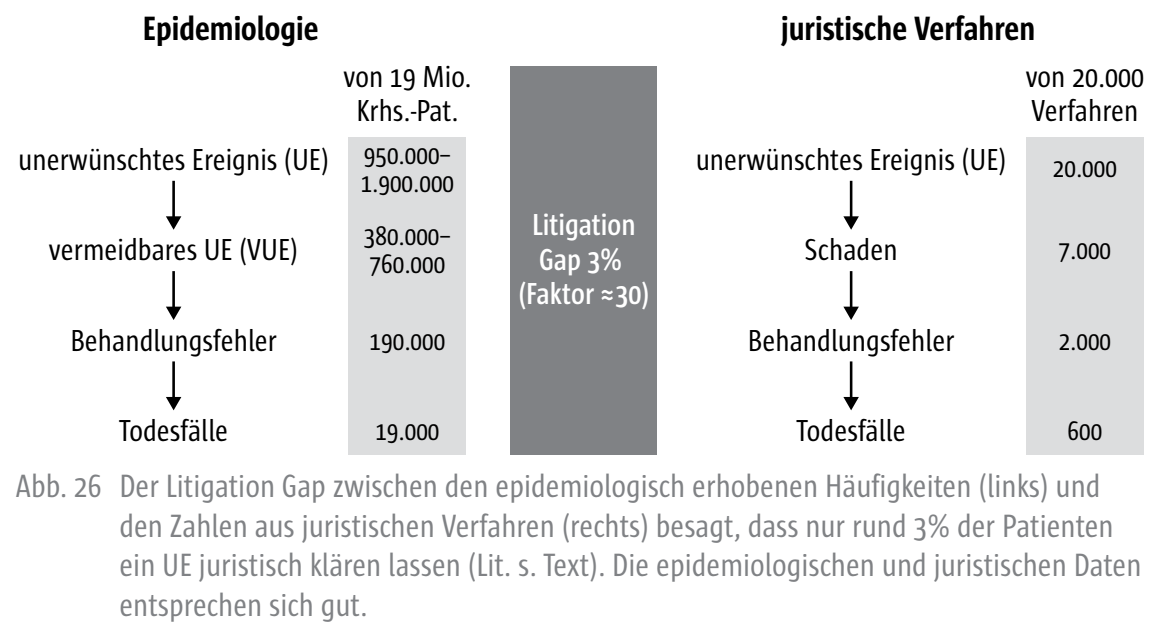


- Als „anderweitige Datenquellen“ kommen natürlich in erster Linie Erhebungen zu nosokomialen Infektionen in Frage, vor allem auch, weil hier langjährig etablierte Erhebungsinstrumente vorliegen (s. Kap. 3.2.5). Das Robert Koch-Institut gab für das Jahr 2006 in Deutschland 400.000 bis 600.000 nosokomiale Infektionen an, die bei 7.500 bis 15.00o Patienten die Todesursache darstellen (Gastmeier et al. 2008). Geht man davon aus, dass davon 1/3 vermeidbar gewesen wären, kommt man auf 2.500-5.00o vermeidbare Todesfälle durch nosokomiale Infektionen, eine Zahl, die mit der Gesamtzahl von 19.0oo Todesfällen durch VUE gut zu vereinbaren ist, denn es handelt sich hier ja nur um eine einzige Gruppe von UE. Eine weitere Gruppe bilden die unerwünschten Arzneimittelereignisse (UAE), die mindestens eine gleich große vermeidbare Mortalität aufweisen (Ebbesen et al. 2001, Pirmohamed et al. 2004, Lazarou et al. 1998). Zur postoperativen Sterblichkeit wurde in einer internationalen Studie (European Surgical Outcome Study) für Deutschland eine Rate von 2,5\% aller operierten Patienten berichtet (europaweit 4,0\%); einen Anteil von 40\% vermeidbarer Ereignisse vorausgesetzt (nicht berichtet), würde dies einer vermeidbaren Sterblichkeit von immerhin etwas mehr als 1\% entsprechen (Pearse et al. 2012). Die postoperative Sterblichkeit in der internationalen „Safe Surgery Saves Lives Study“ der WHO lag bei 1,5\%, auch hier kann man eine Rate zur vermeidbaren Sterblichkeit indirekt ableiten, die um o,5\% liegen dürfte (Haynes et al. 2009). Wenn man all diese Zahlen zusammen zur Kenntnis nimmt (und wichtige Gebiete wie z.B. Stürze, Adverse Medical Device Events (AMDEs) etc. sind ja noch gar nicht mitgerechnet), kann man die vermeidbare Sterblichkeit von $0,1 \%$, die vom Review des APS abgeleitet wurde, kaum als übertrieben bezeichnen; im Grunde handelt es sich um eine sehr konservative Schätzung.

- Ein dritter Weg zur Plausibilisierung ist (alle Welt redet von Patientenerfahrungen als Basis für Patient-Related Outcome Measures) die Befragung und die Einschätzung der Patienten. In einer Serie internationaler Erhebungen des Commonwealth Funds gaben 23\%, 12\%, 19\% und 16\% der Patienten an, sie oder ihre Angehörige hätten bei der medizinischen Behandlung einen „Fehler“ bemerkt (Schoen et al. 2005, 2007, 2009, 2011). In einer anderen Untersuchung gaben $42 \%$ der Befragten (und $35 \%$ befragter Ärzte!) an, bei sich oder den Angehörigen einen „Fehler“ bemerkt zu haben (Blendon et al. 2002). Es gibt hier zwar keine direkten Zahlen zur Sterblichkeit, die Angaben zeigen aber, dass Patienten durchaus ein zutreffendes Bild über die Wirklichkeit haben - ein wichtiges Argument dagegen, man würde durch Veröffentlichung der Zahlen oder durch ein offenes institutionelles Umgehen mit unerwünschten Ereignissen die Öffentlichkeit bzw. Patienten ,verschrecken“, das Gegenteil ist der Fall: man nimmt die Erfahrungen der Patienten ernst, der wichtigste Schritt zur Herstellung einer adäquaten Kommunikation. 
Die hier referierten Zahlen stellen nur einen sehr kleinen Ausschnitt aus der umfangreichen Literatur zu (vermeidbaren) unerwünschten Ereignissen und zur Sterblichkeit dar (vgl. außerdem Häufigkeit von Dekubitalulzera in Kap. 2.4.6). Man muss hinzufügen, dass diese Zahlen das Problem wahrscheinlich immer noch unterschätzen. Wenn man Studien heranzieht, die genauere Erhebungsmethoden (z.B. die begleitende Beobachtung durch geschultes Personal) verwenden, kommt man nämlich auf deutlich höhere Werte (vgl. Andrews et al. 1997). Um ein Beispiel zu nennen: wir sagen, dass in Deutschland Seiten- und Eingriffsverwechselungen, eines der sog. neverevents, nur sehr selten (maximal 1oo Fälle pro Jahr) vorkommen. Im sog. Minnesota Report in den USA werden aber 50 wrong site surgeries pro Woche (!) berichtet, bezogen auf die dreimal größere Bevölkerung der USA würden das bei uns 17 Fällen pro Woche oder goo Fällen im Jahr entsprechen (Chassin 2013). Wir müssen davon ausgehen, dass wir uns nach wie vor nur mit der Spitze des Eisbergs beschäftigen - denn das eigentliche Problem tritt erst bei der näheren Betrachtung der Sachlage und außerdem der Betrachtung von besonders ,verletzlichen" Patientengruppen zutage, z.B. älteren und chronisch erkrankten Patienten (s. folgendes Kap.).

\section{Zusammenfassung:}

Zwischen 5 und 10\% der Patienten im Krankenhaus erleiden ein unerwünschtes Ereignis (behandlungsbedingt, negativer Outcome, unbeabsichtigt), zwischen 2 und $4 \%$ ein vermeidbares unerwünschtes Ereignisse (auf Fehler zurückgehend), um $1 \%$ einen Behandlungsfehler (juristischer Begriff: mangelnde Sorgfalt), und 0,1\% beträgt die vermeidbare Sterblichkeit. Bezogen auf rund 19 Mill. Krankenhausfälle pro Jahr in Deutschland (damals 17. Mill.) ergeben sich 950.000 bis 1,9 Mio. unerwünschte Ereignisse, 380.000 bis 760.000 vermeidbare unerwünschte Ereignisse, ca. 190.000 Behandlungsfehler (juristischer, kein epidemiologischer Begriff) und ca. 19.000 vermeidbare Todesfälle. Diese Zahlen entsprechen dem in den Jahren 2006 und 2008 vom Aktionsbündnis Patientensicherheit durchgeführten Systematischen Review an zuletzt 241 Studien auf der Basis von 44.842 Veröffentlichungen, der auch vom Sachverständigenrat übernommen wurde. Die Zahlen entsprechen anderen Systematischen Review, die international angefertigt worden sind, bzw. liegen unterhalb den dortigen Angaben. Häufigkeiten z.B. zu nosokomialen Infektionen (s. Kap. 3.2.5), unerwünschten Arzneimittelereignissen oder zur postoperativen Sterblichkeit in großen internationalen Studien können genauso zur Plausibilisierung herangezogen werden wie Patientenbefragungen. Die Zahlen sind in Deutschland in der letzten Zeit wieder in Frage gestellt worden, wobei fälschlicherweise nicht zwischen epidemiologischen Erhebungen und juristischen Datenquellen (Schiedsstellen, Haftpflichtversicherer) unterschieden wurde. Der sog. Litigation Gap besagt, dass nur 3\% der Patienten ein unerwünschtes Ereignis klären lassen, über den Faktor 30 sind die Zahlen problemlos miteinander vereinbar. 


\subsubsection{Weiterer Handlungsbedarf; Arzneimittel im Alter, Malpractice Crisis}

Am Anfang der Beschäftigung mit der Thematik Patientensicherheit stehen immer die seltenen, schweren Einzelereignisse katastrophalen Ausmaßes (Patientenverwechselungen, Rechts-Links-Verwechselungen etc.; vgl. Schrappe 2013). Man kann mit Recht sagen, dass es das Verdienst der „Patientensicherheitsbewegung" - von einer Bewegung kann man in den Ländern, in denen dieses Thema bislang auf die Tagesordnung kam, durchaus sprechen ist und bleibt, diese Einzelereignisse, die einem „die Sprache verschlagen“, einer Analyse und Diskussion, somit einer Prävention zugänglich zu machen. Allerdings ist die Zahl dieser never events endlich, nach einiger Zeit sind alle Szenarien wie die versehentliche intrathekale Gabe von Methotrexat etc. aufgearbeitet.

Zu diesem Zeitpunkt stellt sich regelmäßig die Frage: wie weiter?, Patientensicherheit wird normal, es wird zu Risikomanagement, Qualitätsmanagement, Steuerung, und die Thematik wendet sich den „normaleren“, d.h. zwar häufigeren, dafür aber nicht mehr derartig schwerwiegenden Ereignissen zu (s. Abb. 27, hier Themen unter II und auch III). Kurzum: aus Patientenverwechselung wird sekundäres Nierenversagen nach Kontrastmittel. Dieses unerwünschte Ereignis ist auch schwerwiegend, aber war schon zuvor Gegenstand eines umsichtigen Qualitätsmanagements (oder hätte es sein müssen).

Es bleiben die Instrumente, die root cause analysis von Komplikationen, die Prozessanalyse, die generierenden Datenerfassungen, mit denen man versucht, der „blinden Flecken“ Herr zu werden (s. Kap. 2.2). In erster Linie handelt es

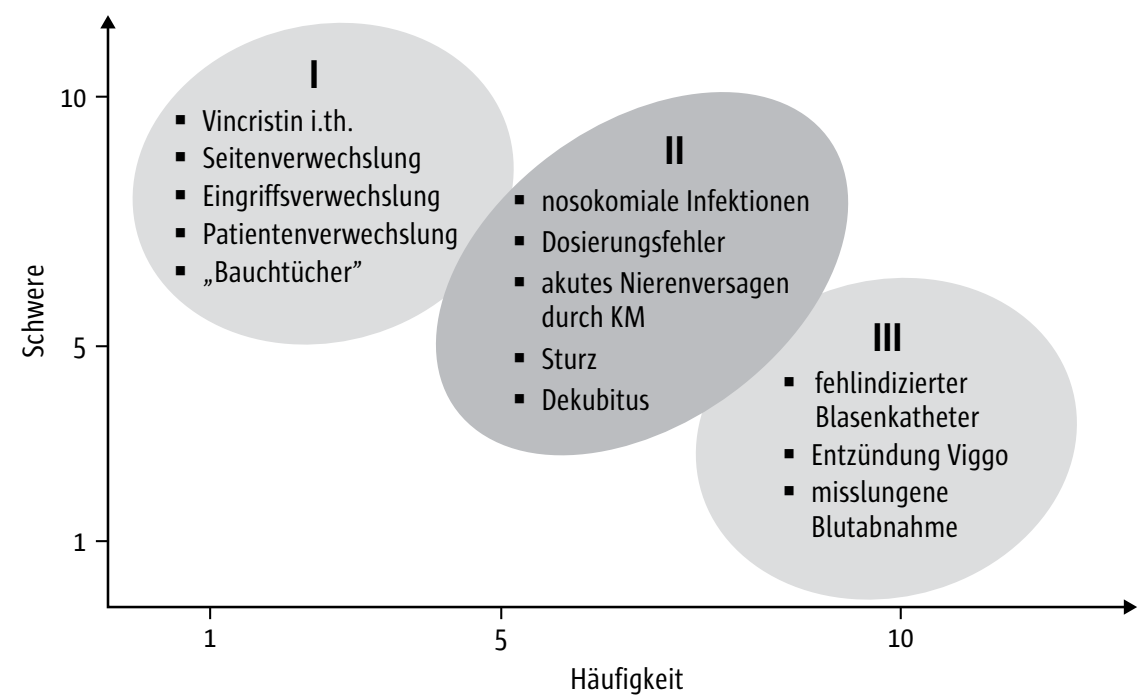

Abb. 27 Patientensicherheit - die Aufgaben verschieben sich nach Bearbeitung der schweren never events (oben links) zu den häufigeren Ereignissen (Mittelfeld). 
sich bei diesen Instrumenten um Critical Incident Reporting Systeme (CIRS), Morbidity-Mortality-Konferenzen, Analyse von Patientenbeschwerden, Analyse von Obduktionsberichten in Abgleich mit den klinischen Diagnosen etc., alles Instrumente, die nicht zur quantitativen Erfassung von Ereignissen geeignet sind, wohl aber zur Erweiterung des Erkenntnishorizontes und als Ausgangspunkt der Analyse - denn man kann nicht analysieren, was man nicht kennt.

Eine andere Richtung, in die sich die Thematik Patientensicherheit bewegen wird und muss, ist die Beschäftigung mit Patientengruppen, die einem besonderen Risiko ausgesetzt sind. Im Sinnzusammenhang mit der weiteren Entwicklung des Gesundheitssystems (demographischer Wandel, Alterung, Multimorbidität) sind hier vor allem ältere Patienten mit Mehrfacherkrankungen zu nennen, die besonders unter Koordinationsmängeln der Versorgung, unter Stürzen und unter unerwünschten Ereignissen im Krankenhaus leiden. Eine ganz zentrale Rolle spielen dabei ihre sprichwörtlich medikamentenüberfüllten Nachtischschubladen - eine Metapher für die weit verbreitete Polypharmakotherapie im Alter über 6o Jahre (gute Übersicht bei Berthold und Steinhagen-Thiessen 2009). Paradoxerweise sind gerade die (eigentlich auf eine Verbesserung der Therapie ausgerichteten) Leitlinien nicht ganz unschuldig an dieser Situation, denn bei mehrfach erkrankten älteren Patienten führt die peinlich genaue Befolgung aller Leitlinien für jede Einzelne ihrer Erkrankungen genau zur Gabe dieser hohen Zahl von Medikamenten, die dann eine unübersehbar große Zahl von unerwünschten Arzneimittelwirkungen und -interaktionen zur Folge haben. Die aus der „Urzeit“ der Leitlinienentwicklung stammende Ansicht, dass Leitlinien lediglich einen „Korridor“ für die Behandlung beschreiben, der unter bestimmten Umständen (z.B. multiple Erkrankungen) sogar verlassen werden muss (!), könnte aktueller nicht sein.

Im konkreten Fall ist es immer wieder die fälschliche Annahme, die unerwünschte Wirkung einer Medikation sei eine neue Erkrankung und somit Anlass für eine neuerliche medikamentöse Therapie, so dass schrittweise immer mehr Medikamente zusammenkommen. Die wichtigsten Fehleinschätzungen betreffen die im Alter meist eingeschränkte Nierenfunktion, über die die meisten Medikamente ausgeschieden werden, und der portosystemische Umgehungskreislauf der Leber (z.B. bei Leberzirrhose), der die Verstoffwechselung bzw. den Abbau der Medikamente verhindert. Die Polypharmakotherapie im Alter führt zu vielfältigen negativen Folgen für die Patienten: Stürze, Immobilisierung, Desorientierung, Depression, Nieren-, Blutbild- und Leberschäden.

Bereits in den 8oer-Jahren des letzten Jahrhunderts hat man begonnen, Empfehlungen zur Vermeidung von unerwünschten Arzneimittelereignissen im Alter zu erarbeiten. Am bekanntesten ist die sog. Beers-Liste (Beers et al. 1991, Beers 1997), die jedoch nur schwer auf die deutschen Verhältnisse zu übertragen war. In Deutschland wurde im Zusammenhang mit der Diskussion um die Arzneimitteltherapiesicherheit (AMTS, s.a. Aktionsplan Arzneimittel- 
therapiesicherheit des Bundesministeriums für Gesundheit) die sog. PriscusListe entwickelt (priscus - altehrwürdig (lat.); s. Holt et al. 2010). Aber auch hier ist die Implementierung und Umsetzung das Problem, ein Sechstel der Notaufnahmepatienten im Alter über 65 Jahre $(16,6 \%)$ nimmt bei der stationären Aufnahme weiterhin mindestens ein Priscus-Medikament ein (Dormann et al. 2013), seit 2008 (21,7\%) hat sich die Situation nicht durchgreifend verbessert (2012: 18,9\%; s. Linder et al. 2014).

Der Handlungsbedarf im Bereich Patientensicherheit ist also noch erheblich, setzt aber eine detaillierte Beschäftigung mit spezifischen Konstellationen voraus und bedarf spezifischer Strategien zur Implementierung. Grundsätzlich müssen für die Patientensicherheit die gleichen Qualitätsperspektiven beachtet werden wie allgemein beim Thema Qualität (s. Kap. 1.3), d.h. auch Probleme, die hinsichtlich des Nutzens von Behandlungsmethoden auftreten (negativer Nettonutzen), verdienen Aufmerksamkeit. Der Sachverständigenrat hat dies z.B. für das Thema Medizinproduktesicherheit erst kürzlich dargestellt (SVR 2014).

Der Handlungsbedarf ergibt sich zusätzlich und in verstärktem Ausmaß durch die Situation auf dem Haftpflichtversicherungsmarkt. Zum Jahreswechsel 2012/2013 haben sich zwei große Versicherungen, Westfälische Provinzial und Zürich, aus dem Markt für Krankenhaushaftpflichtversicherungen zurückgezogen, so dass nur noch vier Anbieter übrig bleiben (Petry und Brabow 2013). Es wird vermutet, dass wenn keine durchgreifende Änderungen der Schadenssummen (es geht nicht nur um die Zahl der juristisch aufgearbeiteten Behandlungsfehler, sondern auch um die gestiegenen Zahlungen pro Fall) zustande kommen (sei es durch ein capping, d.h. eine gesetzliche Beschränkung der Schadensersatzforderungen), die Krankenhäuser bald nicht mehr in der Lage sein werden, die Haftpflichtversicherung zu finanzieren oder überhaupt einen Anbieter zu finden (Petry und Grabow 2013). Bereits zwischen 2010 und 2013 sind die Prämien für 8o,4\% der Krankenhäuser gestiegen, 79,35 der Krankenhäuser, die eine neue Versicherung abgeschlossen haben, mussten Prämiensteigerungen hinnehmen, und zwischen 2010 und 2013 wurde der Vertrag bei 20,7\% der Krankenhäuser durch die Versicherung gekündigt (Offermanns et al. 2013). Die Auseinandersetzungen um die Haftpflichtversicherungen der freiberuflich tätigen Hebammen in den ersten Monaten des Jahres 2014 gehen in die gleiche Richtung.

Die Situation beginnt einer malpractice crisis zu gleichen, einer durch Unterversorgung gekennzeichneten Zugangsbeschränkung, die dadurch entsteht, dass Krankenhäuser (und andere Einrichtungen) Leistungen nicht mehr anbieten können, weil sie sich nicht mehr haftpflichtversichern können (s. Kap. 3.2.1, vgl. Schwerpunktheft von Health Affairs Nr. 1/2014, weiterhin Mello et al. 2003, Mohr 2000, Runciman et al. 2003, Studdert et al. 2004). Die Situation war in den letzten Jahrzehnten in den USA besonders drastisch, teilweise waren in manchen Bundesstaaten die Versorgung für bestimmte Fachdisziplinen 
nicht mehr aufrechtzuerhalten (Dranove und Grona 2005). Deutlich kam der Widerspruch zwischen der punitiven Philosophie (Prämien müssen hoch sein, damit die Krankenhäuser reagieren) und der präventiven Haltung (Risikoprävention ist der richtige Ansatz) zum Vorschein (Runciman et al. 2003, Studdert et al. 2004, Wachter 2005). Die Haftpflichtversicherungsindustrie gerät in einen strukturellen Widerspruch zur Patientensicherheitsbewegung, weil sie falls sie weiter im Haftpflichtversicherungsgeschäft bleiben will - gegenüber ihren stakeholdern gezwungen ist, die Situation positiver darzustellen als sie ist (Gausmann 2014). Unterschiedliche Mittel werden zur Abhilfe diskutiert, reichend von der direkten finanziellen Unterstützung für die Prämienzahlungen, so wie es im Fall der Hebammen in Deutschland geschehen ist (und sofort Begehrlichkeiten an anderer Stelle geweckt hat) bis hin zur Deckelung der Schadensersatzzahlungen (Übersicht bei Mello und Gallagher 2010).

\section{Zusammenfassung:}

Patientensicherheit wird „normales“ Risiko- und Qualitätsmanagement, aber spezielle Patientengruppen mit hohem Risiko (z.B. ältere Patienten) und eine drohende Versicherungskrise (malpractice crisis) müssen im Fokus bleiben. Der demographische Wandel rückt ältere Patienten mit Mehrfacherkrankungen in den Mittelpunkt, die besonders unter Koordinationsmängeln der Versorgung, unter Stürzen, unter unerwünschten Ereignissen im Krankenhaus und der weit verbreiteten Polypharmakotherapie leiden. Obwohl es Empfehlungen zur Vermeidung von unerwünschten Arzneimittelereignissen gibt (z.B. Priscus-Liste), erhalten rund $20 \%$ der älteren Patienten ein Medikament, das nach PriscusListe nicht empfohlen wird. Eine Versicherungskrise wurde zuletzt bei der Hebammenversorgung diskutiert. Es ist durchaus möglich, dass in Zukunft Krankenhäuser (und andere Einrichtungen) Leistungen nicht mehr anbieten können, weil sie keine Haftpflichtversicherung erhalten.

\subsection{Perspektive der Patienten}

Was zählt aus der Sicht der Patienten? Alles, würde man sagen, Ergebnisse, Prozesse, Sicherheit ... nicht umsonst erlebt man immer wieder, dass die „Sicht der Patienten in den Mittelpunkt gestellt wird“ (s. Kap. 1.3). Patientenbefragungen zeigen, dass Patienten sehr zutreffende Ansichten über die Häufigkeit von unerwünschten Ereignissen und Schäden haben (s. Kap. 3.3.3.2), diese aber sicherlich nicht selbst erleben möchten. Patienten haben auch in Deutschland, stellt man erstaunt fest, Probleme mit dem Zugang zur Versorgung (s. Kap. 3.2.1). Defizite z.B. im Bereich der Schmerztherapie haben immer noch ein erschreckendes Ausmaß (s. Kap. 3.3.2). Indikatoren auf der Basis von Patientenerfahrungen, so wie sie jetzt auch im deutschen Gesundheitswesen entwickelt werden sollen (s. FQWG vom 5.6.2014 mit den Anforderungen an das Institut für Qualitätssicherung und Transparenz im Gesundheitswesen in $\$ 137 \mathrm{a}$ [neu], Abs. 3), sind international bereits in Gebrauch (Patienten 
Reported Outcome Measures, s. Kap. 2.4.7). Patienten wird eine wichtige Rolle im Qualitätswettbewerb zugewiesen (s. Kap. 4.4), und mittels Transparenz werden ihnen hierfür Qualitätsinformationen zur Verfügung gestellt (s. Kap. 5.3, Public Reporting). Patientenvertreter spielen auch in den Institutionen des Gesundheitswesens eine immer wichtigere Rolle (zu den gesetzlichen Regelungen s. Kap. 7.4).

Die wichtigsten sicherheitsrelevanten Faktoren, die Patienten in Befragungen angeben, betreffen jedoch die Themen Kommunikation und Koordination. Bei Entlassung aus dem Krankenhaus haben 23\% (Schoen et al. 2005) und 29\% der befragten Patienten aus Deutschland (Schoen et al. 2009) keine Instruktionen, für 50\% (Schoen et al. 2005) bzw. 35\% (Schoen et al. 2009) wurden keine Arrangements getroffen. Befunde fehlen in 11\% (Schoen et al. 2005), 8\% (Schoen et al. 2007) bzw. 12\% (Schoen et al. 2009). 32\% bzw. 17\% der Patienten haben beobachtet, dass Fachärzten keine Anamnese der Hausärzte vorlag bzw. der Hausarzt keinen Bericht vom Facharzt erhalten hat (Schoen et al. 2009).

Auch international ist gut untersucht, dass der Übergang von einer Institution (bzw. Sektor) in die andere für das Auftreten von unerwünschten Ereignissen besonders relevant ist. In einer monozentrischen holländischen Studie erlitten in den ersten vier Wochen nach Entlassung aus dem Krankenhaus 25\% der Patienten ein unerwünschtes Ereignis (Marang-van de Mheen et al. 2008). In einer anderen Studie erlitten 6\% der Patienten nach Entlassung aus der Notaufnahme eines Krankenhauses (ohne stationäre Aufnahme) ein unerwünschtes Ereignis (Forster et al. 2007).

Bezüglich der Koordination der Behandlung spielt die Medikation eine besonders wichtige Rolle. Die Arzneimitteltherapie ist ein sehr differenzierter Vorgang, weist eine hohe Rate von unerwünschten Arzneimittelwirkungen und Interaktionen auf und stellt hohe Anforderungen an die organisatorische Umsetzung (Arzneimittelversorgung, Verabreichung der Arzneimittel etc.). Eine klassische Konstellation, die recht gut untersucht ist, stellt die stationäre Aufnahme wegen (schwerer) unerwünschter Arzneimittelereignisse bzw. -wirkungen dar (z.B. Bhalla et al. 2003, Pirmohammed et al. 2004, Pouyanne et al. 200o). Wie in Kapitel 2.1 bereits dargestellt, muss man in Deutschland von jährlich knapp 400.00o Patienten ausgehen, die wegen unerwünschter Arzneimittelereignisse stationär aufgenommen werden müssen (Schneeweiss et al. 2002). Ältere Patienten sind besonders betroffen (Mannesse et al. 2000).

Aus diesem Grunde ist es vor allem bei Patienten über 6o Jahre sinnvoll, die Medikation so oft wie möglich zu überprüfen, um den Kenntnisstand der Behandelnden zu sichern, damit mögliche unerwünschte Arzneimittelereignisse zu verhindern bzw. das akute klinische Bild auf das Vorliegen eines solchen Ereignisses zu überprüfen ist, um Interaktionen zu identifizieren und um die Medikation auf die Bedürfnisse des Patienten anzupassen. Dieser Prozess, der auch als medication reconciliation bezeichnet wird (Nassaralla et al. 2007), war grundsätzlich als Bestandteil des „Aktionsplanes zur Verbesserung der Arznei- 
mitteltherapiesicherheit in Deutschland“ des Bundesministeriums für Gesundheit vorgesehen (zuletzt in der Fassung 2013-2015, s. www.ap-amts.de). Er ist aber immer noch nicht umgesetzt, weil die Beteiligten die Kostenfrage in den Vordergrund stellen, obwohl die Medikamentenanamnese als selbstverständlicher und basaler Teil der ärztlichen Tätigkeit anzusehen ist, der durch die gängige Vergütung finanziert sein sollte. Hier sind zweifelsohne die Patienten und Patientenverbände sowie die politische Ebene gefragt.

\section{Zusammenfassung:}

Patientenerfahrungen und Patientenvertreter spielen mittlerweile eine wichtige Rolle, als Patient-Reported Outcome Measures, als Akteure im sog. Qualitätswettbewerb und als Vertreter in den Gremien des Gesundheitswesens. Jedoch sind gerade aus der Patientenperspektive weiterhin gravierende Mängel feststellbar. Bei Entlassung aus dem Krankenhaus haben ein Viertel der Patienten keine Instruktionen, für bis zur Hälfte der Patienten wurden keinerlei Arrangements getroffen. Befunde fehlen bei $10 \%$ der Patienten, zwischen $32 \%$ bzw. 17\% der Patienten beobachten, dass Fachärzten keine Anamnese der Hausärzte vorlag bzw. der Hausarzt keinen Bericht vom Facharzt erhalten hat. Es ist besonders der Übergang zwischen den verschiedenen Institutionen und Versorgungsektoren, der für das Auftreten von unerwünschten Ereignissen besonders relevant ist. In einer monozentrischen holländischen Studie erlitten in den ersten vier Wochen nach Entlassung aus dem Krankenhaus 25\% der Patienten ein unerwünschtes Ereignis. Die regelmäßige Überprüfung der Medikation (sog. medication reconciliation) ist immer noch nicht umgesetzt.

\subsection{Professionen}

Die Professionen im Gesundheitswesen sind im Zusammenhang mit ihrer ausgeprägten intrinsischen Motivation sehr am Erhalt ihrer Autonomie interessiert (zum Begriff des Professionalismus s. Kap. 8.3.1 und 8.4.3). Da sowohl für Expertenorganisationen (professional bureaucracies, s. Kap. 8.2) als auch für komplexe Systeme (s. Kap. 8.3) das Umgehen mit Paradoxa und Unsicherheit nicht ungewohnt ist (zum Begriff der „intrinsischen Unsicherheit“" s. Kap. 8.3.3 und 9.5), wird die externe Forderung nach Qualitätsverbesserung und Verbesserung der Patientensicherheit als inadäquate Einmischung erlebt und nur schwer akzeptiert. In Wertschätzung der professionellen Autonomie hat man den Standesorganisationen weitgehende Aufgaben im Bereich der Qualitätssicherung, Aus- und Fortbildung und auch der Patientensicherheit (Schiedsstellen auf der Ebene der Landesärztekammern) eingeräumt. Wenn jedoch offensichtliche Sachverhalte wie z.B. der Unterschied zwischen epidemiologischen und juristischen Daten zur Häufigkeit von unerwünschten Ereignissen nicht zur Kenntnis genommen werden (s. Kap. 3.3.3.2), kommen doch ernsthafte Zweifel auf, inwieweit diese Verantwortung in vollem Umfang übernommen wird. 
Letztlich ist auch die Institution des Gemeinsamen Bundesausschusses eine letztlich professionell dominierte Struktur. Im Jahr 2005 wurde die Zuständigkeit für die Thematik Qualität vom Bundesministerium für Gesundheit auf den GBA übertragen (s. Kap. 7.2.2). Gerade im Zusammenhang mit dem neuen Institut für Qualitätssicherung und Transparenz im Gesundheitswesen ( $\$ 137$ a SGBV [neu]) ist zu hoffen, dass durch die Öffentlichkeit, die der Entscheidungsfindung zur Fragen der Qualität und Sicherheit dort zukommt, eine kohärentere Vorgehensweise eingeschlagen wird. Das Umgehen mit der Thematik Patientensicherheit Anfang des letzten Jahrzehnts war diesbezüglich sehr ermutigend. Es wäre sehr wünschenswert, dass aus der professionellen Haltung ein Engagement z.B. in Richtung einer deutschen Choosing Wisely-Kampagne (s. Kap. 3.3.1) Raum greift.

\subsection{Institutionen}

Institutionen spielen in der Erbringung und Umsetzung von Qualität und Patientensicherheit eine erhebliche Rolle, und zwar in mehrerlei Hinsicht:

n die institutionelle Perspektive ist eine der sechs Qualitätsperspektiven (s. Kap. 1.3),

- Einrichtungen im Gesundheitswesen reagieren als Expertenorganisationen nicht linear und vorhersagbar auf externe Qualitäts- und Sicherheitserwartungen (s. Kap. 8.2),

- sie sind ihrerseits integriert in das komplexe System des Gesundheitswesens, das ebenfalls schwer beeinflussbar und steuerbar ist (s. Kap. 8.3),

- sowohl Expertenorganisationen als auch komplexen Systemen ist eine hochgradige Toleranz gegenüber Unsicherheit und paradoxen Situationen zu Eigen (s. intrinsische Unsicherheit, s. Kap. 8.3.3 und 9.5),

v in den gesetzlichen Crundlagen zum Qualitäts- und Risikomanagements wurde der institutionellen Umsetzung viel Raum gegeben (s. Kap. 7.6),

- die organisatorische Komponente (Aufgaben- und Strukturgestaltung bis hin zu Organisationslernen und Organisationskultur, s. Kap. 8.4) ist eine der wichtigsten konstituierenden Elemente von Qualität und Sicherheit, und

- haftungsrechtlich ist das Organisationsverschulden eines der wichtigsten Faktoren für juristische Auseinandersetzungen, insbesondere für die Frage der Beweislasterleichterung (s. Kap. 1.3).

Obwohl das Bürgerliche Gesetzbuch den Begriff des Organisationsverschuldens nicht kennt (Katzenmeier 2007) und der Begriff auch im Patientenrechtegesetz vom 13.1.2013 bzw. in $\mathbb{S}$ 630a ff. BGB nicht genannt ist (wenngleich dort z.B. die Aufklärungs- und Dokumentationspflichten ausführlich beschrieben werden), ist dieser Begriff von großer haftungsrechtlicher Bedeutung. Krankenhausträger, Krankenhausleitung und Leitende Krankenhausärzte müssen z.B. Zuständigkeiten und Verantwortlichkeiten im ärztlichen 
Dienst genau regeln, Mitarbeiter müssen sorgfältig ausgewählt und überwacht werden, die medizinischen Standards müssen gewährleistet und die Sicherheit muß garantiert werden (Haier und Bergmann 2013). Organisation und Koordination des Behandlungsgeschehens werden grundsätzlich als voll beherrschbar angesehen und führen daher zu einer Beweislasterleichterung für den Patienten (Katzenmeier 2007).

Eine besondere Verantwortung besteht hinsichtlich der Dienstplangestaltung und Personalausstattung. Die Verantwortlichen müssen vermeiden, dass Personal wegen Übermüdung nicht mehr in der Lage ist, seine Tätigkeit verantwortungsvoll auszuführen (Jansen 2007). Diese Problematik ist in den letzten Jahren durch neuere Untersuchungen noch entscheidender geworden, die einen Zusammenhang zwischen Personalausstattung bzw. Dienstplanregelungen (sog. staffing) und Komplikationen zu belegen scheinen (Yaghoubian et al. 2008, Aiken et al. 2014).

Organisatorisch bedingte unerwünschte Ereignisse und Ursachen von Schäden sind häufig, wenn Komplikationen genau analysiert werden (root cause analysis). man unterscheidet Ursachen, die in der Arbeitsumgebung liegen, von Team-Faktoren und mangelnder Aufgabenspezifizierung (z.B. Dean et al. 2002). Leider sind populationsbezogene Zahlen über unerwünschte Ereignisse organisatorischer Provenienz in der Literatur selten, soweit sie nicht nur auf einzelnen Prozessen oder Fachgebieten beruhen (z.B. Transfusionsmedizin, s. Zeiler und Kretschmer 2005). Wie maßgeblich organisatorische Defizite den Ablauf in den Kliniken auch weiterhin bestimmen, zeigen neuere Untersuchungen zur OP-Organisation; in 20-40\% der Patienten kommt es morgens zu einer Verzögerung von im Mittel (je nach Fachgebiet) von 14 bis 21 Minuten (Schuster et al. 2013). Wenn man in einer überschlägigen Abschätzung davon ausgeht, dass bei rund to Mill. Operationen pro Jahr in deutschen Krankenhäusern und 2 Mill. „ersten OPs“ in einem Drittel davon (70o.ooo Operationen) eine Verspätung von 15 Minuten auftritt, dann kommt man auf ca. 10 Mill. Verspätungsminuten allein bei der ersten OP am Morgen. Als Überleitung auf das nächste Kapitel: Bei Zugrundelegung der Annahme, dass eine OP-Minute $10 €$ an Kosten verursacht, würden durch mangelnde OP-Auslastung nur bezüglich der ersten morgendlichen OP zusätzliche Kosten von rund 100 Mill. € entstehen. Wohlgemerkt sind hier die Kosten, die durch weitere Verschiebungen, die durch die Verspätung der (neuralgischen) ersten OP am Tag entstehen, sowie die Verschiebung bzw. den Ausfall von Operationen noch gar nicht mit berücksichtigt.

\subsection{Wissenschaft}

Aus wissenschaftlicher Perspektive bieten Qualität und Sicherheit eine große Zahl von Themen, die vor allem auch die Umsetzung der Forschungsergebnisse aus der Grundlagen- und klinisch-evaluativen Forschung in die reale Ver- 
sorgung betreffen (s. Kap. 1.3, Schrappe und Scriba 2011B). Qualitäts- und Sicherheitsforschung sind am ehesten in der Bereich der Versorgungsforschung zu verorten (Schrappe 2011D, 2011E). Allerdings ist durch die fast ausschließlich Grundlagenorientierung der deutschen Medizinischen Fakultäten die Situation eingetreten, dass der Großteil der entsprechenden Studien im angloamerikanischen Bereich veröffentlicht wird, wie auch das Literaturverzeichnis des vorliegenden Gutachtens zeigt. Hinzu kommt, dass durch die rigide Trennung von Krankenversorgung auf der einen Seite und Forschung und Lehre auf der anderen Seite an der Universitätskliniken bzw. Fakultäten die Themen Qualität und Sicherheit von wissenschaftlicher Seite oft in den „unwissenschaftlichen“ Bereich abgedrängt werden (,ist doch Krankenversorgung“). Andererseits ist allerdings nicht zu verkennen, dass insbesondere im Bereich der öffentlichen Forschungsförderung diese Aspekte mittlerweile immer mehr und aktiv gefordert und gefördert werden.

Neben der efficacy/effectiveness-Problematik (Transfer in die Alltagsversorgung) ist wissenschaftlich vor allem die Überführung von linearen Fragestellungen und Studienergebnissen in komplexe Konzepte von Interesse. Die Einführung der Evidence-Based Medicine als Instrument der Validitätsüberprüfung von Ergebnissen biomedizinischer Studien war ein großer Fortschritt, heutzutage müssen aber auch die Umsetzungsbedingungen und die Kontextfaktoren wissenschaftlich valide belegt werden, z.T. unter Zuhilfenahme sozialwissenschaftlicher Methoden. Auch hier muss Nachvollziehbarkeit und Validität gewährleistet sein, deshalb ist die Arbeit an den Memoranden des Deutschen Netzwerkes Versorgungsforschung (DNVF) von so großer Wichtigkeit. International ist der Begriff Evidence-Based Health Care geläufig, um „die EBM der Versorgungsforschung “ zu charakterisieren (Cochrane Collaboration 2008). Die Problematik besteht in der Integration von biomedizinischen Ergebnissen und organisationstheoretischen sowie verhaltenspsychologischen und ökonomischen Konzepten, um die relevanten Qualitätsdefizite zu erkennen, zu analysieren und Maßnahmen zur Behebung dieser Defizite zu implementieren.

Inhaltlich stehen die großen populationsbezogenen Fragen wie nosokomiale Infektionen und Antibiotikaresistenzentwicklung im Vordergrund, so wie sie auch in den vorangegangenen Kapiteln dargestellt wurden. Zweifelsohne handelt es sich dabei um komplexe Probleme, die wiederum durch komplexe Instrumente angegangen werden müssen (zum Begriff der „doppelten Komplexität“"s. Kap. 8.3.2). Ein Erfolg ist dabei nur dann zu erreichen, wenn die wissenschaftliche Evaluation auf der Basis eines theoretischen Konzepte geschieht, so wie es international vorliegt (Crossing the Quality Chasm des IOM [2001] in den USA und The NHS Outcome Framework 2013/2014 in Großbritannien [NHS 2013B]) und in diesem Gutachten entwickelt und dargestellt werden soll. Erst ein solches Konzept erlaubt es, Fragestellungen zu identifizieren, die Rahmenbedingungen für den Einsatz der Instrumente zu beschreiben und vor allem Voraussagen über die zu beobachtenden Effekte zu treffen, so dass die Evaluation sinnvoll geplant werden kann. 


\subsection{Kosten durch Qualitäts- und Patientensicherheitsdefizite}

Zu den Kosten durch Qualitätsdefizite liegt eine große Zahl von Untersuchungen vor. Den Schwerpunkt bilden dabei die nosokomialen Infektionen, unerwünschte Arzneimittelereignisse und unerwünschte Ereignisse aus dem Bereich Patientensicherheit. Die Analysen zu den nosokomialen Infektionen gehen bis in die goer-Jahre des letzten Jahrhunderts zurück. Nach Schätzungen der Centers of Disease Control (CDC) in den USA wurden bereits damals auf der Basis von jährlich 2 Mio. nosokomialen Infektionen Kosten von knapp \$6oo für die Harnwegsinfektionen, knapp \$ 3.000 für Wundinfektionen, \$ 5.0oo für Pneumonien und zwischen $\$ 3.000$ und \$ 40.000 für die nosokomiale Sepsis berechnet (Jarvis 1996). Neuere Studien in den USA ergeben zusätzliche Kosten von knapp \$ 7.000 bei vermuteter und über \$15.00o für eine bestätigte nosokomiale Infektion (Roberts et al. 2003), wobei das Spektrum je nach Art der Stationen und Organmanifestationen erheblich schwankt (Chen et al. 2009).

Die Berechnung der inkrementellen Kosten von nosokomialen Infektionen und anderen Komplikationen beziehen sich fast immer auf die direkten Kosten; indirekte (z.B. Verdienstausfall) und intangible (nicht-materielle) Kosten werden nur selten mit einbezogen. Die gängige Approximation für die zusätzlichen Kosten im Krankenhaus besteht in der Verlängerung der stationären Verweildauer (Zhan und Miller 2003, Wagenbach 2010). Schon früh wurde nachgewiesen, dass die Komorbiditätsadjustierung des DRG-Systems nicht für eine Kostendeckung ausreicht (Haley et al. 1997), was wohl auch sinnvoll ist, soweit man nosokomiale Infektionen in einem gewissen Teil für vermeidbar hält (z.B. durch Händedesinfektion). Im Rahmen der Non-Payment for NonPerformance Policy (s. Kap. 4.4; Graves et al. 2008, Pronovost et al. 2008) wurden für eine Auswahl von nosokomialen Infektionen diese zusätzlichen Zahlungen einbehalten.

Mittlerweile hat die Diskussion um die Kosten nosokomialer Infektionen auch Deutschland erreicht (Kersting et al. 2014), wobei hier nicht so viele Studien hierzu vorliegen. Eine nosokomiale Harnwegsinfektion, die nosokomiale Infektion mit dem geringsten Schweregrad, schlägt mit ca. $1.000 €$ zu Buche (Vonberg et al. 2008). In einer Case-Control-Studie wurden die zusätzlichen Kosten einer nosokomialen Pneumonie durch MRSA auf über $17.000 €$ beziffert, interessanterweise um 5.00o € höher liegend als eine Pneumonie durch methicillinsensible Staphylokokken (MSSA, Ott et al. 2010).

Es sind einige Studien für unerwünschte Arzneimittelereignisse vorhanden. Eine amerikanische Analyse an Patientenakten erbrachte zusätzliche Kosten für vermeidbare Ereignisse von \$ 3.500 pro Fall bzw. einer Verlängerung der Liegezeit von 3,4 Tagen (Hug et al. 2012). Eine spanische Analyse, die auf einem Literaturreview beruht, schließt darauf, dass auf Spanien jährliche Kosten von zwischen 469 und 790 Mill. $€$ allein durch unerwünschte Arzneimittelereignisse zukommen, 91 Mill. davon vermeidbar (Anonymous 2008). 
Weniger Untersuchungen liegen allgemein für unerwünschte Ereignisse aller Art vor (Oevreveit und Toll 2009). Ein Systematischer Review des Kanadischen Patient Safety Institute erbrachte für das Jahr 2009/2010 inkrementelle Kosten von \$ $1.1 \mathrm{Mrd}$., \$ 400 Mill. davon verursacht durch vermeidbare unerwünschte Ereignisse (Etchells et al. 2012). Eine Modellierung auf der Basis von Haftpflichtversicherungsdaten aus den USA erbrachte sogar jährliche Kosten von \$17,1 Mrd. (van den Bos et al. 2011). Eine niederländische Analyse, die sich nicht auf eine systematische Literatursuche, sondern auf die direkte Datenerhebung an knapp 8.ooo Patienten stützt, erbringt für das Jahr 2004 Kosten durch vermeidbare unerwünschte Ereignisse von 161 Mill. €, 2,3\% aller stationären Aufnahmen und 3\% aller Liegetage gingen auf vermeidbare unerwünschte Ereignisse zurück (entsprechend 1\% des nationalen Gesundheitsbudgets; Hoonhout et al. 2009).

Je nach Grunderkrankung konnte in den USA eine Verlängerung der Liegezeit von bis zu 11 Tagen bei der postoperativen Sepsis nachgewiesen werden (Zhan und Miller 2003), im letzteren Fall entsprechend zusätzlichen Kosten von \$57.727 bei einer zuzuordnenden Mortalität von 22\%. An der zweiten Stelle stand die postoperative Wunddehiszenz mit einer Verlängerung der Verweildauer von knapp 9,5 Tagen, einer zusätzlichen Mortalität von 9,6\% und Kosten von $\$ 40.323$.

Auf der Basis der hier genannten Studien kann eine Kostenabschätzung für nosokomiale Infektionen und unerwünschte Ereignisse in Deutschland nur näherungsweise versucht werden. Interessant sind ja die vermeidbaren Infektionen bzw. Ereignisse. Geht man also von 5.000 vermeidbaren Infektionen pro Jahr (Gastmeier et al. 2010, s. Kap. 3.3.2) und Kosten pro Infektion von $5.000 €$ aus, kann man die zusätzlich entstehenden Kosten vermeidbarer (!) Infektionen auf 25 Mill. € schätzen. Diese Schätzung ist sehr vorsichtig und gibt die untere Grenze der Streuung wieder. Wenn man nämlich in einem anderen Zugang nur die zusätzlich notwendigen Liegetage zugrundelegt (ca. 1 Mill. Liegetage pro Jahr, s. Gastmeier et al. 2005) und pro Liegetag $500 €$ veranschlagt, kommt man zu zusätzlichen jährlichen Kosten von 500 Mill. € bzw. ca. 170 Mill. € durch vermeidbare Infektionen. Diese Zahl liegt unter den Angaben aus Großbritannien, wo von jährlichen Kosten von 1,25 Mrd. € ausgegangen wird, ein Drittel davon entfallend auf vermeidbare nosokomiale Infektionen (400 Mill. €; HCPAC 2000).

Auf der Basis des Systematischen Reviews des Aktionsbündnis Patientensicherheit aus dem Jahr 2008 (APS 2008) wurden 33 Studien identifiziert, die eine Verweildauerverlängerung als Folge unerwünschter Ereignisse untersuchten und eine Veränderung zwischen 0,5 und 20,1 Tagen berichteten, in 4 Studien mit Risikoadjustierung zwischen 2,3 und 8,2 Tagen (Wagenbach 2010). Legt man also für Deutschland eine durchschnittliche Verweildauerverlängerung von 5 Tagen à $500 €$ zugrunde, würden bei 570.000 vermeidbaren unerwünschten Ereignissen pro Jahr (3\%, s. Kap. 3.3.3.2) zusätzliche und ver- 
meidbare Kosten von 1,425 Mrd. € auftreten. Diese Angabe liegt im Bereich der oben angeführten niederländische Analyse, denn für Deutschland entspräche dies zusätzlichen 440.000 vermeidbaren Aufnahmen (2,3\% aller Aufnahmen) bzw. bei Fallkosten von $3.000 €$ jährlichen Mehrkosten von 1,3 Mrd. € (Hoonhout et al. 2009). Die Kanadische Analyse liegt in der Größenordnung der Angaben für Deutschland (inkrementelle Kosten von \$ 400 Mill. durch vermeidbare unerwünschte Ereignisse (Etchells et al. 2012)), ebenso die spanische Analyse (91 Mill. € durch unerwünschte Arzneimittelereignisse, Anonymous 2008). Die Analyse auf der Basis von Haftpflichtversicherungsdaten aus den USA mit jährlichen Kosten von \$17,1 Mrd. liegt natürlich weitaus höher und zeigt die Streuung, mit der man rechnen muss (van den Bos et al. 2011). Wie oben angemerkt, handelt es sich hierbei nur um direkte Kosten, Produktionsbzw. Verdienstausfälle und intangible Kosten sind nicht mit einbezogen.

Zusammenfasssung: (1) Nosokomiale Infektionen: In Deutschland liegen die zusätzlichen jährlichen Kosten durch vermeidbare nosokomiale Infektionen zwischen 25 Mill. $€$ und 170 Mill. $€$. In Großbritannien rechnet man mit 400 Mill. $€$. (2) Vermeidbare unerwünschte Arzneimittelereignisse kosten $\$ 3.500$ pro Fall bzw. sind für eine Verlängerung der Liegezeit um 3,4 Tage verantwortlich. In Spanien rechnet man mit jährlichen Kosten durch vermeidbare unerwünschte Arzneimittelereignisse von 91 Mill. €. (3) Vermeidbare unerwünschte Ereignisse aller Art verursachen nach dem Systematischem Review des Aktionsbündnis Patientensicherheit 2008 ( 33 Studien enthielten Kostendaten) eine Verlängerung der Liegezeit zwischen 0,5 und 20,1 Tagen (4 Studien mit Risikoadjustierung zwischen 2,3 und 8,2 Tagen). Entsprechend würden vermeidbare unerwünschte Ereignisse in Deutschland zusätzliche Kosten von 1,425 Mrd. € verursachen. Auf der Basis einer niederländischen Studie errechnen sich 1,3 Mrd. €. Eine Kanadische Analyse zeigt inkrementelle Kosten von $\$ 400$ Mill. durch vermeidbare unerwünschte Ereignisse, eine Modellierung auf Basis von Haftpflichtversicherungsdaten aus den USA erbrachte sogar jährliche Kosten von \$17,1 Mrd. 